\title{
Matricial Representations of Certain Finitely Presented Groups Generated by Order-2 Generators and Their Applications
}

\author{
Ryan Golden*, Ilwoo Cho \\ Department of Mathematics, Saint Ambrose University, Davenport, IA \\ Students: goldenryanm@sau.edu* \\ Mentor: choilwoo@sau.edu
}

\begin{abstract}
In this paper, we study matricial representations of certain finitely presented groups $\Gamma_{N}^{2}$ with $N$-generators of order-2. As an application, we consider a group algebra $\mathcal{A}_{2}$ of $\Gamma_{2}^{2}$, under our representations. Specifically, we characterize the inverses $g^{-1}$ of all group elements $g$ in $\Gamma_{2}^{2}$, in terms of matrices in the group algebra $\mathcal{A}_{2}$. From the study of this characterization, we realize there are close relations between the trace of the radial operator of $\mathcal{A}_{2}$, and the Lucas numbers appearing in the Lucas triangle.
\end{abstract}

\section{KEYWORDS}

Matricial Representation; Group Presentation; Group Algebras; Lucas Numbers; Lucas Triangle; Finitely Presented Group; Group Relations; Free Probability

\section{INTRODUCTION}

In this paper, we consider certain finite-dimensional representations $\left(\mathbb{C}^{n}, \alpha^{n}\right)$ of a finitely presented group:

$$
\Gamma_{N}^{2} \stackrel{\text { def }}{=}\left\langle\left\{x_{1}, \ldots, x_{N}\right\},\left\{x_{1}^{2}=\ldots=x_{N}^{2}\right\}\right\rangle
$$

Equation 1.

where the generators $x_{1}, \ldots, x_{N}$ are indeterminants. Precisely, the algebraic structure $\Gamma_{N}^{2}$ is the group generated by $N$ generators $x_{1}, \ldots, x_{N}$ equipped with a noncommutative binary operation (Denoted by adjacency), satisfying

$$
x_{1}^{2}=x_{2}^{2}=\ldots=x_{N}^{2}=e_{N},
$$

Equation 2.

where $e_{N}$ is the group-identity of $\Gamma_{N}^{2}$. One may understand a group $\Gamma_{N}^{2}$ as the quotient group,

$$
\Gamma_{N}^{2} \stackrel{\text { Group }}{=} \mathcal{F}_{N} /\left\{g_{j}^{2}=e: g_{j} \text { are generators of } \mathcal{F}_{N}\right\},
$$

Equation 3.

where $\mathcal{F}_{N}$ is the noncommutative (non-reduced) free group $\left\langle\left\{g_{j}\right\}_{j=1}^{N}\right\rangle$ with $N$ generators $g_{1}, \ldots, g_{N}$, where $e$ is the group-

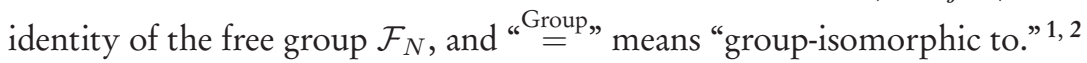

By construction, all elements $g$ of the group $\Gamma_{N}^{2}$ are of the form,

$$
g=x_{i_{1}}^{k_{1}} x_{i_{2}}^{k_{2}} \ldots x_{i_{n}}^{k_{n}}, \text { for some } i_{1}, \ldots, i_{n} \in\{1, \ldots, N\} \text {, and } k_{1}, \ldots, k_{n} \in \mathbb{Z},
$$

Equation 4.

as non-reduced words in $\left\{x_{1}, \ldots, x_{N}\right\}$, where $x_{j}^{-1}$ means the group-inverse of $x_{j}$, and hence, $x_{j}^{-k}$ means $\left(x_{j}^{-1}\right)^{k}$, for all $k \in \mathbb{N}$, for all $j=1, \ldots N$. In this paper, for an arbitrarily fixed $N \in \mathbb{N}$, we establish and study certain $n$-dimensional Hilbert-space representations $\left(\mathbb{C}^{n}, \alpha^{n}\right)$ of the groups $\Gamma_{N}^{2}$, for all $n \in \mathbb{N} \backslash\{1\}$. Under our representations, each element $g$ of $\Gamma_{N}^{2}$ is understood as a matrix $A_{g}$ acting on $\mathbb{C}^{n}$, for $n \in \mathbb{N}$. Moreover, if $g=x_{i_{1}}^{k_{1}} \ldots x_{i_{l}}^{k_{l}}$ as in Equation 4 (as a non-reduced word in $\Gamma_{N}^{2}$ ), then there exists corresponding matrices $A_{i_{1}}, \ldots, A_{i_{l}}$, such that $A_{g}=A_{i_{1}}^{k_{1}} \ldots A_{i_{l}}^{k_{l}} \in M_{n}(\mathbb{C}$ ) (as a reduced word in $M_{n}(\mathbb{C})$ ), where $M_{n}(\mathbb{C})$ is the matricial algebra consisting of all $(n \times n)$-matrices, for $n \in \mathbb{N}$. 


\section{Motivation}

Here we justify our interest in the groups $\Gamma_{N}^{2}$, for $N \in \mathbb{N}$.

Free probability is a branch of operator algebra. By considering free-distributional data, one can establish operator-valued noncommutative probability theory on (topological or pure-algebraic) algebras as in classical probability theory. ${ }^{3,4}$ Also, such free-probabilistic data allow structure theorems for given algebras under free product, determined by given linear functionals. Here, the independence of classical probability is replaced by freeness. There are two approaches in free probability theory: Voiculescu's original analytic approach, ${ }^{4}$ and Speicher's combinatorial approach. ${ }^{3}$

The author, Cho, has considered connections between operator algebra theory and Hecke-algebraic number theory via free probability, to provide tools for studying number-theoretic results with operator-algebraic techniques, and vice versa, by establishing certain representational and operator-theoretic models from combinatorial free probability settings. ${ }^{3}$

In a series of research papers, Cho considered the free-probabilistic representations of the Hecke Algebras $\mathcal{H}\left(G_{p}\right)$ generated by the generalized linear $((2 \times 2)$-matricial $)$ groups, $G_{p}=G L_{2}\left(\mathbb{Q}_{p}\right)$, over the $p$-adic number field $\mathbb{Q}_{p}$ for prime $p .{ }^{1,2,5}$

Cho and Gillespie established free probability models of certain subalgebras $\mathcal{H}_{Y_{p}}$ of the Hecke algebras $\mathcal{H}\left(G_{p}\right)$ by defining suitable linear functionals on $\mathcal{H}\left(G_{p}\right)$. In particular, they constructed free-probabilistic structures preserving numbertheoretic data from $\mathcal{H}_{Y_{p}} \cdot{ }^{5}$

Cho extended certain free-probabilistic models of $\mathcal{H}_{Y_{p}}$ to those of $\mathcal{H}\left(G_{p}\right)$ fully, for all primes $p{ }^{2,6}$ On such models, the $C^{*}$-algebras $\mathbb{H}\left(G_{p}\right)$ were constructed by realizing elements of $\mathcal{H}\left(G_{p}\right)$ as operators under free-probabilistic representations of $\mathcal{H}\left(G_{p}\right)$, and the operator-theoretic properties of generating operators of $\mathbb{H}\left(G_{p}\right)$ were studied there.

By studying certain types of partial isometries of $\mathbb{H}\left(G_{p}\right)$, Cho obtained the embedded non-abelian multiplicative groups $G_{N}$ of $\mathbb{H}\left(G_{p}\right)$ generated by $N$-many partial isometries. ${ }^{1}$ In particular, it was shown that

$$
G_{N} \stackrel{\text { Group }}{=} \Gamma_{N}^{2}, \text { for all } N \in \mathbb{N},
$$

Equation 5.

where $\Gamma_{N}^{2}$ is in the sense of Equation 1, satisfying Equation 3. To study detailed algebraic properties of $G_{N}$ in $\mathcal{H}\left(G_{p}\right)$, and to investigate operator-algebraic properties of $C^{*}\left(G_{N}\right)$ in $\mathbb{H}\left(G_{p}\right)$, he used the isomorphic group $\Gamma_{N}^{2}$ of Equation 1, and the corresponding group $C^{*}$-algebra: $C^{*}\left(\Gamma_{N}^{2}\right)$.

In this paper, we study the groups $\Gamma_{N}^{2}$ of Equation 1 pure-algebraically (independent from our sources). ${ }^{1-3}$

\section{Overview}

In this paper, we concentrate on studying the groups $\Gamma_{N}^{2}$ of Equation 1 independently. The main purpose is to establish suitable Hilbert-space representations of $\Gamma_{N}^{2}$ other than those established in our sources. ${ }^{1}$ Fundamentally, we construct finite-dimensional Hilbert-space representations $\left(\mathbb{C}^{n}, \alpha^{n}\right)$ of $\Gamma_{N}^{2}$, for all $n \in \mathbb{N} \backslash\{1\}$.

Under our representation $\left(\mathbb{C}^{n}, \alpha^{n}\right)$ for $n \in \mathbb{N}$, each group element $g \in \Gamma_{N}^{2}$ is understood as a matrix $A_{g}$ in the matricial algebra $M_{n}(\mathbb{C})$. To study an algebraic object $g \in \Gamma_{N}^{2}$, we will investigate functional properties of the corresponding matrices $A_{g} \in M_{n}(\mathbb{C})$, for $n \in \mathbb{N}$.

As application, we consider a group algebra $\mathcal{A}_{2}$ of $\Gamma_{2}^{2}$, under our representations. Specifically, we characterize the inverses $g^{-1}$ of all group elements $g$ in $\Gamma_{2}^{2}$, in terms of matrices in the group algebra $\mathcal{A}_{2}$ generated by $\Gamma_{2}^{2}$.

From the study of this characterization, we show that there are close relations between the trace of the powers $T^{n}$ of the radial operator $T$ of $\mathcal{A}_{2}$, and the Lucas numbers in the Lucas triangle. 


\section{FINITE-DIMENSIONAL REPRESENTATIONS OF $\Gamma_{N}^{2}$}

In this section, we establish finite-dimensional Hilbert-space representations of the finitely presented groups $\Gamma_{N}^{2}$ with $N$ generators of order-2 in the sense of Equation 1: $\Gamma_{N}^{2}=\left\langle\left\{x_{j}\right\}_{j=1}^{N},\left\{x_{j}^{2}=e_{N}\right\}_{j=1}^{N}\right\rangle$, where $x_{1}, \ldots, x_{j}$ are noncommutative indeterminants (as generators), and $e_{N}$ is the group identity of $\Gamma_{N}^{2}$. Fix $N \in \mathbb{N}$ throughout this section and fix the corresponding group $\Gamma_{N}^{2}$. Denote by $I_{N}$ the identity matrix in $M_{N}(\mathbb{C})$

\section{A Matrix Group $\mathfrak{M}_{2}^{2}(N)$}

Let $M_{2}(\mathbb{C})$ be the $(2 \times 2)$-matricial algebra, and let $\mathbb{C}^{\times}=\mathbb{C} \backslash\{0\}$. Denote by $\mathbb{C} \times \mathbb{C}^{\times}$the Cartesian product of $\mathbb{C}^{\text {and }} \mathbb{C}^{\times}$. Define a function

$$
A_{2}: \mathbb{C} \times \mathbb{C}^{\times} \rightarrow M_{2}(\mathbb{C}) \text { by } A_{2}(a, b)=\left(\begin{array}{cc}
a & b \\
\frac{1-a^{2}}{b} & -a
\end{array}\right)
$$

Equation 6.

for all $(a, b) \in \mathbb{C} \times \mathbb{C}^{\times}$.

Lemma 1. Let $A$ be the map in the sense of Equation 6. Then

$$
\left(A_{2}(a, b)\right)^{2}=I_{2}
$$

Equation 7.

for all $(a, b) \in \mathbb{C} \times \mathbb{C}^{\times}$.

Proof. The proof is straightforward computation.

It is also easy to verify that:

$$
\begin{aligned}
& A_{2}\left(a_{1}, b_{1}\right) A_{2}\left(a_{2}, b_{2}\right)=\left(\begin{array}{cc}
\frac{a_{1} a_{2} b_{2}+b_{1}-b_{1} a_{2}^{2}}{b_{2}} & a_{1} b_{2}-b_{1} a_{2} \\
\frac{a_{2} b_{2}-a_{2} b_{2} a_{1}^{2}-a_{1} b_{1}+a_{1} b_{1} a_{2}^{2}}{b_{1} b_{2}} & \frac{b_{2}-b_{2} a_{1}^{2}+a_{1} a_{2} b_{1}}{b_{1}}
\end{array}\right) \\
& A_{2}\left(a_{2}, b_{2}\right) A_{2}\left(a_{1}, b_{1}\right)=\left(\begin{array}{cc}
\frac{b_{2}-b_{2} a_{1}^{2}+a_{1} a_{2} b_{1}}{b_{1}} & b_{1} a_{2}-a_{1} b_{2} \\
\frac{-a_{2} b_{2}+a_{2} b_{2} a_{1}^{2}+a_{1} b_{1}-a_{1} b_{1} a_{2}^{2}}{b_{1} b_{2}} & \frac{a_{1} a_{2} b_{2}+b_{1}-b_{1} a_{2}^{2}}{b_{2}}
\end{array}\right)
\end{aligned}
$$

Equation 8.

and hence,

$$
A_{2}\left(a_{1}, b_{1}\right) A_{2}\left(a_{2}, b_{2}\right) \neq A_{2}\left(a_{2}, b_{2}\right) A_{2}\left(a_{1}, b_{1}\right)
$$

Equation 9.

in general, for $\left(a_{k}, b_{k}\right) \in \mathbb{C} \times \mathbb{C}^{\times}$and $k=1$, 2. In particular, whenever $\left(a_{k}, b_{k}\right) \in \mathbb{C}^{\times} \times \mathbb{C}^{\times}$, for $k=1,2$, and the pairs $\left(a_{1}, b_{1}\right)$ and $\left(a_{2}, b_{2}\right)$ are distinct in $\mathbb{C}^{\times} \times \mathbb{C}^{\times}$, the above noncommutativity Equation $\mathbf{9}$ always holds. Therefore, the image, $\left\{A_{2}(a, b):(a, b) \in \mathbb{C}^{\times} \times \mathbb{C}^{\times}\right\}$, forms a noncommutative family in $M_{2}(\mathbb{C})$, by Equation 9.

Now, take distinct pairs,

$$
\left(a_{k}, b_{k}\right) \in \mathbb{C}^{\times} \times \mathbb{C}^{\times}, \text {for } k=1, \ldots, N .
$$

Equation 10.

One can construct the corresponding matrices,

$$
A_{2}\left(a_{k}, b_{k}\right) \in M_{2}(\mathbb{C}), \text { for } k=1, \ldots, N,
$$

Equation 11.

by Equation 6. Under the matrix multiplication on $M_{2}(\mathbb{C})$, let's construct the multiplicative subgroup $\mathfrak{M}_{2}^{2}(N)$ of $M_{2}(\mathbb{C})$ as

$$
\mathfrak{M}_{2}^{2}(N)=\left\langle\left\{A_{2}\left(a_{k}, b_{k}\right)\right\}_{k=1}^{N}\right\rangle
$$

Equation 12.

generated by $A_{2}\left(a_{k}, b_{k}\right)$, for all $k=1, \ldots, N$. Then all elements of $\mathfrak{M}_{2}^{2}(N)$ are the $(2 \times 2)$-matrices in $M_{2}(\mathbb{C})$, satisfying

$$
\left(A_{2}\left(a_{k}, b_{k}\right)\right)^{2}=I_{2} \in \mathfrak{M}_{2}^{2}(N),
$$

Equation 13.

for all $k=1, \ldots, N$, by Equation 7 . 
Definition 1. Let $\mathfrak{M}_{2}^{2}(N)$ be the multiplicative subgroup of $M_{2}(\mathbb{C})$, as defined in Equation 12. We call $\mathfrak{M}_{2}^{2}(N)$ the 2-dimensional, order-2, $N$-generator (sub-)group (of $M_{2}(\mathbb{C})$ ).

One obtains the following algebraic characterization.

Theorem 2. Let $\mathfrak{M}_{2}^{2}(N)$ be a 2-dimensional, order-2, $N$-generator group in $M_{2}(\mathbb{C})$. Then the groups $\mathfrak{M}_{2}^{2}(N)$ and the group $\Gamma_{N}^{2}$ of Equation 1 are isomorphic:

$$
\mathfrak{M}_{2}^{2}(N) \stackrel{\text { Group }}{=} \Gamma_{N}^{2}
$$

Equation 14.

Proof. Let $\mathfrak{M}_{2}^{2}(N)$ be a 2-dimensional, order-2, $N$-generator group in $M_{2}(\mathbb{C})$. Then, by Equation 13, each generator $A_{2}\left(a_{k}, b_{k}\right)$ satisfies $\left(A_{2}\left(a_{k}, b_{k}\right)\right)^{2}=I_{2}$, for all $k=1, \ldots, N$.

Since $\left(a_{k}, b_{k}\right)$ are taken from $\mathbb{C}^{\times} \times \mathbb{C}^{\times}$, the generators $\left\{A_{2}\left(a_{k}, b_{k}\right)\right\}_{k=1}^{N}$ form a noncommutative family in $M_{2}(\mathbb{C})$, by Equation 9.

Observe that there does not exist an $n$-tuple $\left(j_{1}, \ldots, j_{n}\right)$ of distinct elements $j_{1}, \ldots, j_{n}$ in $\{1, \ldots, N\}$ such that

$$
\prod_{l=1}^{n} A_{2}\left(a_{k_{j_{l}}}, b_{k_{j_{l}}}\right)=A_{2}\left(a_{k_{j_{0}}}, b_{k_{j_{0}}}\right) \text {, or } \prod_{l=1}^{n} A_{2}\left(a_{k_{j_{l}}}, b_{k_{j_{l}}}\right)=I_{2}, \quad \text { Equation } 15 .
$$

for some $j_{0} \in\{1, \ldots, N\}$, by Equations 6 and $\mathbf{9}$. These observations show that $\mathfrak{M}_{2}^{2}(N) \stackrel{\text { Group }}{=} \mathcal{F}_{N} / \mathcal{R}_{N}$, where $\mathcal{F}_{N}$ is the noncommutative free group $\left\langle\left\{g_{j}\right\}_{j=1}^{N}\right\rangle$ with $N$-generators $g_{1}, \ldots, g_{N}$, and $\mathcal{R}_{N}$ is the relator set, $\mathcal{R}_{N}=\left\{g_{j}^{2}=e\right\}_{j=1}^{N}$.

Recall that the group $\Gamma_{N}^{2}$ of Equation 1 is group-isomorphic to the quotient group $\mathcal{F}_{N} / \mathcal{R}_{N}$, by Equation 3. Therefore, one has that

$$
\mathfrak{M}_{2}^{2}(N) \stackrel{\text { Group }}{=} \mathcal{F}_{N} / \mathcal{R}_{N} \stackrel{\text { Group }}{=} \Gamma_{N}^{2} \text {. }
$$

Equation 16.

The 2-dimensional, order-2, $N$-generator group $\mathfrak{M}_{2}^{2}(N)$ is group isomorphic to the group $\Gamma_{N}^{2}$ of Equation 1 .

The groups $\mathfrak{M}_{2}^{2}(N)$ and $\Gamma_{N}^{2}$ are isomorphic to each other. Equivalently, there exists a group isomorphism $\alpha^{2}: \Gamma_{N}^{2} \rightarrow$ $\mathfrak{M}_{2}^{2}(N)$, satisfying

$$
\alpha^{2}\left(x_{j}\right)=A_{2}\left(a_{j}, b_{j}\right), \text { for all } j=1, \ldots, N .
$$

Equation 17.

The above structure theorem provides a 2-dimensional Hilbert-space representation $\left(\mathbb{C}^{2}, \alpha^{2}\right)$ of $\Gamma_{N}^{2}$.

Theorem 3. There exists a 2-dimensional Hilbert-space representation $\left(\mathbb{C}^{2}, \alpha^{2}\right)$ of $\Gamma_{N}^{2}$. Additionally, $\alpha^{2}(g)$ acting on $\mathbb{C}^{2}$ are in the sense of Equation 17, for all $g \in \Gamma_{N}^{2}$.

Proof. Since $\alpha^{2}$ of Equation 17 is a generator-preserving group-isomorphism from $\Gamma_{N}^{2}$ to $\mathfrak{M}_{2}^{2}(N)$, it satisfies

$$
\begin{gathered}
\alpha^{2}\left(g_{1} g_{2}\right)=\alpha^{2}\left(g_{1}\right) \alpha^{2}\left(g_{2}\right) \in \mathfrak{M}_{2}^{2}(N) \text { for all } g_{1}, g_{2} \in \Gamma_{N}^{2} \\
\alpha^{2}\left(g^{-1}\right)=\left(\alpha^{2}(g)\right)^{-1} \in \mathfrak{M}_{2}^{2}(N) \text { for all } g \in \Gamma_{N}^{2} .
\end{gathered}
$$

Since $\mathfrak{M}_{2}^{2}(N) \subseteq M_{2}(\mathbb{C})$, the matrices $\alpha^{2}(g)$ are acting on $\mathbb{C}^{2}$, for all $g \in \Gamma_{N}^{2}$. Therefore, the pair $\left(\mathbb{C}^{2}, \alpha^{2}\right)$ forms a Hilbertspace representation of $\Gamma_{N}^{2}$. 


\section{Certain Order-2 Matrices in $M_{n}(\mathbb{C})$}

Now, let $n>2$ in $\mathbb{N}$ and consider certain types of matrices in $M_{n}(\mathbb{C})$. In the previous section, we showed that our group $\Gamma_{N}^{2}$ of Equation 1 is group isomorphic to the multiplicative subgroup $\mathfrak{M}_{2}^{2}(N)$ of Equation 12 and hence, we obtain a natural 2-dimensional representation $\left(\mathbb{C}^{2}, \alpha^{2}\right)$ of $\Gamma_{N}^{2}$. In this section, we construct a base-stone to extend the representation $\left(\mathbb{C}^{2}, \alpha^{2}\right)$ to arbitrary $n$-dimensional representations $\left(\mathbb{C}^{n}, \alpha^{n}\right)$ of $\Gamma_{N}^{2}$, for all $n \in \mathbb{N} \backslash\{1\}$. To this end, we fix the matrices $A_{2}(a, b)$, defined in Equation 6, for use as blocks of certain matrices in $M_{n}(\mathbb{C})$. For our main purpose, we always assume that $(a, b) \in \mathbb{C}_{\sim_{1}}^{\times} \times \mathbb{C}^{\times}$, whenever we have matrices $A_{2}(a, b)$ in the following text, as in Equation 10, where $\mathbb{C}_{\sim_{1}}^{\times} \stackrel{\text { def }}{=} \mathbb{C} \backslash\{0,1\}$.

First take $n=3$, and consider a matrix $A_{3}(a, b, c)$ defined to be

$$
A_{3}(a, b, c)=\left(\begin{array}{ccc}
1 & c & \frac{-b c}{1-a} \\
0 & a & b \\
0 & \frac{1-a^{2}}{b} & -a
\end{array}\right) \in M_{3}(\mathbb{C}),
$$

Equation 18.

for some $a, b, c \in \mathbb{C}^{\times}$. This matrix $A_{3}(a, b, c)$ is the block matrix,

$$
A_{3}(a, b, c)=\left(\begin{array}{cc}
(1) & \left(\begin{array}{cc}
c & \frac{-b c}{1-a}
\end{array}\right) \\
\left(\begin{array}{l}
0 \\
0
\end{array}\right) & A_{2}(a, b)
\end{array}\right) \in M_{3}(\mathbb{C}) .
$$

Equation 19.

By straightforward computation, one can obtain

$$
A_{3}(a, b, c)^{2}=I_{3}=\left(\begin{array}{ccc}
1 & 0 & 0 \\
0 & 1 & 0 \\
0 & 0 & 1
\end{array}\right)
$$

Equation 20.

Define a morphism $A_{3}: \mathbb{C}_{\sim_{1}}^{\times} \times \mathbb{C}^{\times} \times \mathbb{C}^{\times} \rightarrow M_{3}(\mathbb{C})$ by

$$
A_{3}(a, b, c)=\left(\begin{array}{cc}
(1) & \left(\begin{array}{cc}
c & \frac{-b c}{1-a}
\end{array}\right) \\
\left(\begin{array}{l}
0 \\
0
\end{array}\right) & A_{2}(a, b)
\end{array}\right) .
$$

Equation 21.

By Equation 20, for any $(a, b, c) \in \mathbb{C}_{\sim_{1}}^{\times} \times \mathbb{C}^{\times} \times \mathbb{C}^{\times}$, we have $\left(A_{3}(a, b, c)\right)^{2}=I_{3}$. Now let $n=4$ and consider a matrix $A_{4}(a, b, c, d)$ defined by

$$
A_{4}(a, b, c, d)=\left(\begin{array}{cccc}
1 & 0 & d & \frac{-b d}{1-a} \\
0 & 1 & c & \frac{-b c}{1-a} \\
0 & 0 & a & b \\
0 & 0 & \frac{1-a^{2}}{b} & -a
\end{array}\right) \in M_{4}(\mathbb{C}),
$$

Equation 22.

for $a, b, c, d \in \mathbb{C}^{\times}$. Similarly, this matrix $A_{4}(a, b, c, d)$ is regarded as the block matrix,

$$
\begin{aligned}
A_{4}(a, b, c, d) & =\left(\begin{array}{ccc}
(1) & \left(\begin{array}{ccc}
0 & d & \frac{-b d}{1-a}
\end{array}\right) \\
\left(\begin{array}{c}
0 \\
0 \\
0
\end{array}\right) & & A_{3}(a, b, c)
\end{array}\right) \\
A_{4} & =\left(\begin{array}{cc}
I_{2} & \left(\begin{array}{cc}
d & \frac{-b d}{1-a} \\
c & \frac{-b c}{1-a} \\
O_{2,2} & A_{2}(a, b)
\end{array}\right) .
\end{array}\right.
\end{aligned}
$$

Equation 23. 
in $M_{4}(\mathbb{C})$, where $O_{n, m}$ is the $(n \times m)$-zero matrix. Then, by the direct computation, one obtains that

$$
A_{4}^{2}=I_{4}
$$

Equation 24.

Similar to Equation 22, we define a morphism $A_{4}: \mathbb{C}_{\sim_{1}}^{\times} \times\left(\mathbb{C}^{\times}\right)^{3} \rightarrow M_{4}(\mathbb{C})$ by

$$
A_{4}(a, b, c, d)=\left(\begin{array}{cc}
I_{2} & Q_{a, b}(c, d) \\
O_{2,2} & A_{2}(a, b)
\end{array}\right),
$$

Equation 25.

where

$$
Q_{a, b}(c, d)=\left(\begin{array}{cc}
d & \frac{-b d}{1-a} \\
c & \frac{-b c}{1-a}
\end{array}\right)
$$

Equation 26.

The image $A_{4}(a, b, c, d)$ is a well-defined matrix in $M_{4}(\mathbb{C})$, by Equations 22 and 23. Moreover, by Equation 24, we have

$$
\left(A_{4}(a, b, c, d)\right)^{2}=I_{4} \in M_{4}(\mathbb{C}) .
$$

Equation 27.

Let's consider one more step: let $n=5$. Similar to the above, we define the following map. $A_{5}: \mathbb{C}_{\sim_{1}}^{\times} \times\left(\mathbb{C}^{\times}\right)^{4} \rightarrow M_{5}(\mathbb{C})$ by

$$
A_{5}\left(a_{1}, \ldots, a_{5}\right)=\left(\begin{array}{cc}
I_{3} & Q_{a_{1}, a_{2}}\left(a_{3}, a_{4}, a_{5}\right) \\
O_{2,3} & A_{2}\left(a_{1}, a_{2}\right)
\end{array}\right),
$$

Equation 28.

for all $\left(a_{1}, \ldots, a_{5}\right) \in\left(\mathbb{C}^{\times}\right)^{5}$, where

$$
Q_{a_{1}, a_{2}}\left(a_{3}, a_{4}, a_{5}\right)=\left(\begin{array}{cc}
a_{5} & \frac{-a_{2} a_{5}}{1-a_{1}} \\
a_{4} & \frac{-a_{2} a_{4}}{1-a_{1}} \\
a_{3} & \frac{-a_{2} a_{3}}{1-a_{1}}
\end{array}\right)
$$

Equivalently,

$$
A_{5}\left(a_{1}, \ldots, a_{5}\right)=\left(\begin{array}{ccccc}
1 & 0 & 0 & a_{5} & \frac{-a_{2} a_{5}}{1-a_{1}} \\
0 & 1 & 0 & a_{4} & \frac{-a_{2} a_{4}}{1-a_{1}} \\
0 & 0 & 1 & a_{3} & \frac{-a_{2} a_{3}}{1-a_{1}} \\
0 & 0 & 0 & a_{1} & a_{2} \\
0 & 0 & 0 & \frac{1-a_{1}^{2}}{a_{2}} & -a_{1}
\end{array}\right) \in M_{5}(\mathbb{C})
$$

Equation 30.

From direct computation, one again obtains that

$$
\left(A_{5}\left(a_{1}, \ldots, a_{5}\right)\right)^{2}=I_{5} .
$$

Equation 31.

Inductively, for $n \geq 3$, we define the following map $A_{n}: \mathbb{C}_{\sim_{1}}^{\times} \times\left(\mathbb{C}^{\times}\right)^{n-1} \rightarrow M_{n}(\mathbb{C})$, by

$$
A_{n}\left(a_{1}, \ldots, a_{n}\right)=\left(\begin{array}{cc}
I_{n-2} & Q_{a_{1}, a_{2}}\left(a_{3}, \ldots, a_{n}\right) \\
O_{2, n-2} & A_{2}\left(a_{1}, a_{2}\right)
\end{array}\right),
$$

Equation 32.

for all $\left(a_{1}, \ldots, a_{n}\right) \in\left(\mathbb{C}^{\times}\right)^{n}$, where $A_{2}\left(a_{1}, a_{2}\right)$ is in the sense of Equation 6, $O_{2, n-2}$ is the $(2 \times(n-2))$-zero matrix, $I_{n-2}$ is the $(n-2) \times(n-2)$ identity matrix, and

$$
Q_{a_{1}, a_{2}}\left(a_{3}, \ldots, a_{n}\right)=\left(\begin{array}{cc}
a_{n} & \frac{-a_{2} a_{n}}{1-a_{1}} \\
\vdots & \vdots \\
a_{4} & \frac{-a_{2} a_{3}}{1-a_{1}} \\
a_{3} & \frac{-a_{2} a_{3}}{1-a_{1}}
\end{array}\right) .
$$

Then we obtain the following computation. 
Theorem 4. Let $A_{n}$ be the morphism Equation 32, and let $A_{n}\left(a_{1}, \ldots, a_{n}\right)$ be the image of $A_{n}$ realized in the matricial algebra $M_{n}(\mathbb{C})$, for fixed $n \geq 3$ in $\mathbb{N}$. Then

$$
\left(A_{n}\left(a_{1}, \ldots, a_{n}\right)\right)^{2}=I_{n},
$$

Equation 34.

the identity matrix of $M_{n}(\mathbb{C})$.

Proof. Let $n \geq 3$ be given in $\mathbb{N}$.

By Equations 20, 27 and 31, we have $\left(A_{k}\left(a_{1}, \ldots, a_{k}\right)\right)^{2}=I_{k} \in M_{k}(\mathbb{C})$, for $k=3,4,5$.

Now, without loss of generality take $n \geq 6$ in $\mathbb{N}$. Then

$$
A_{n}\left(a_{1}, \ldots, a_{n}\right)=\left(\begin{array}{cc}
I_{n-2} & Q_{a_{1}, a_{2}}\left(a_{3}, \ldots, a_{n}\right) \\
O_{2, n-2} & A_{2}\left(a_{1}, a_{2}\right)
\end{array}\right) .
$$

For convenience, we let

$$
\begin{aligned}
& I \stackrel{\text { denote }}{=} I_{n-2} \\
& Q \stackrel{\text { denote }}{=} Q_{a_{1}, a_{2}}\left(a_{3}, \ldots, a_{n}\right) \\
& O \stackrel{\text { denote }}{=} O_{2, n-2} \\
& A \stackrel{\text { denote }}{=} A_{2}\left(a_{1}, a_{2}\right)
\end{aligned}
$$

Equation 36.

So

$$
\left(\begin{array}{ll}
I & Q \\
O & A
\end{array}\right) \stackrel{\text { denote }}{=} A_{n}\left(a_{1}, \ldots, a_{n}\right)
$$

Equation 37.

as a block matrix in $M_{n}(\mathbb{C})$. Then

$$
\begin{aligned}
\left(A_{n}\left(a_{1}, \ldots, a_{n}\right)\right)^{2} & =\left(\begin{array}{cc}
I & Q \\
O & A
\end{array}\right)\left(\begin{array}{cc}
I & Q \\
O & A
\end{array}\right) \\
& =\left(\begin{array}{cc}
I^{2}+Q O & I Q+Q A \\
O I+A O & O Q+A^{2}
\end{array}\right) \\
& =\left(\begin{array}{cc}
I & I Q+Q A \\
O & A^{2}
\end{array}\right) \\
& =\left(\begin{array}{cc}
I & Q+Q A \\
O & I_{2}
\end{array}\right),
\end{aligned}
$$

Equation 38 .

by Equation 7. So, to show Equation 34, it is sufficient to show that $Q+Q A=O_{n-2,2}$. 
Notice that

$$
\begin{aligned}
Q A & =\left(\begin{array}{cc}
a_{n} & \frac{-a_{2} a_{n}}{1-a_{1}} \\
\vdots & \vdots \\
a_{4} & \frac{-a_{2} a_{4}}{1-a_{1}} \\
a_{3} & \frac{-a_{2} a_{3}}{1-a_{1}}
\end{array}\right)\left(\begin{array}{cc}
a_{1} & a_{2} \\
\frac{1-a_{1}^{2}}{a_{2}} & -a_{1}
\end{array}\right) \\
& =\left(\begin{array}{cc}
a_{1} a_{n}+\frac{-a_{2} a_{n}\left(1-a_{1}^{2}\right)}{a_{2}\left(1-a_{1}\right)} & a_{2} a_{n}+\frac{a_{1} a_{2} a_{n}}{1-a_{1}} \\
\vdots & \vdots \\
a_{1} a_{3}+\frac{-a_{2} a_{3}\left(1-a_{1}^{2}\right)}{a_{2}\left(1-a_{1}\right)} & a_{2} a_{3}+\frac{a_{1} a_{2} a_{3}}{1-a_{1}}
\end{array}\right) \\
& =\left(\begin{array}{cc}
-a_{n} & a_{2} a_{n}\left(1+\frac{a_{1}}{1-a_{1}}\right) \\
\vdots & \vdots \\
-a_{3} & a_{2} a_{3}\left(1+\frac{a_{1}}{1-a_{1}}\right)
\end{array}\right) .
\end{aligned}
$$

Equation 39.

Thus,

$$
\begin{aligned}
Q+Q A & =\left(\begin{array}{cc}
a_{n} & \frac{-a_{2} a_{n}}{1-a_{1}} \\
\vdots & \vdots \\
a_{3} & \frac{-a_{2} a_{3}}{1-a_{1}}
\end{array}\right)+\left(\begin{array}{cc}
-a_{n} & a_{2} a_{n}\left(1+\frac{a_{1}}{1-a_{1}}\right) \\
\vdots & \\
-a_{3} & a_{2} a_{3}\left(1+\frac{a_{1}}{1-a_{1}}\right)
\end{array}\right) \\
& =\left(\begin{array}{cc}
0 & a_{2} a_{n}\left(\frac{-1}{1-a_{1}}+1+\frac{a_{1}}{1-a_{1}}\right) \\
\vdots & \vdots \\
0 & a_{2} a_{3}\left(\frac{-1}{1-a_{1}}+1+\frac{a_{1}}{1-a_{1}}\right)
\end{array}\right) \\
& =\left(\begin{array}{cc}
0 & 0 \\
\vdots & \vdots \\
0 & 0
\end{array}\right) \\
& =O_{n-2,2},
\end{aligned}
$$

Equation 40.

So, for any $n \geq 6$,

$$
\left(A_{n}\left(a_{1}, \ldots, a_{n}\right)\right)^{2}=\left(\begin{array}{cc}
I & Q+Q A \\
O & I
\end{array}\right)=\left(\begin{array}{cc}
I & O_{n-2,2} \\
O & I
\end{array}\right)=I_{n} .
$$

Equation 41.

$n$-Dimensional Hilbert-Space Representations of $\Gamma_{N}^{2}$

Fix $N \in \mathbb{N}$ and $n \in \mathbb{N} \backslash\{1\}$. Let $\Gamma_{N}^{2}$ be our finitely presented group as defined in Equation 1. For the fixed $n$, we take the $n$-tuples

$$
W_{k}=\left(a_{k, 1}, \ldots, a_{k, n}\right) \in \mathbb{C}_{\sim 1}^{\times} \times\left(\mathbb{C}^{\times}\right)^{n-1},
$$

Equation 42.

for $k=1, \ldots, N$, and assume that $W_{1}, \ldots, W_{N}$ are mutually distinct.

Observation and Notation As we assumed above, let $W_{1}, \ldots, W_{N}$ be mutually distinct in $\mathbb{C}_{\sim}^{\times} \times\left(\mathbb{C}^{\times}\right)^{n-1}$. For our purposes, one may further assume that these mutually distinct $n$-tuples satisfy $W_{i} \neq W_{j}$ in the sense that $a_{i, l} \neq a_{j, l} \in \mathbb{C}^{\times}$, for all $l=1, \ldots, N$. If the $n$-tuples $W_{1}, \ldots, W_{N}$ satisfy the above stronger condition than the mutually-distinctness, we say they are strongly mutually distinct. Define the corresponding matrices,

$$
X_{k} \stackrel{\text { denote }}{=} A_{n}\left(W_{k}\right)=\left(\begin{array}{cc}
I_{n-2} & Q_{a_{k, 1}, a_{k, 2}}\left(a_{k, 3}, \ldots, a_{k, n}\right) \\
O_{2, n-2} & A_{2}\left(a_{k, 1}, a_{k, 2}\right)
\end{array}\right),
$$

Equation 43. 
as in Equation 32, where $W_{k}$ are strongly mutually distinct $n$-tuples of Equation $\mathbf{4 2}$, for all $k=1, \ldots, N$.

Then, one can create the multiplicative subgroup $\mathfrak{M}_{n}^{2}(N)$ of $M_{n}(\mathbb{C})$ from the (reduced) free group generated by $\left\{X_{1}, \ldots\right.$, $\left.X_{N}\right\}: \mathfrak{M}_{n}^{2}(N)=\left\langle\left\{X_{j}\right\}_{j=1}^{N}\right\rangle \in M_{n}(\mathbb{C})$.

Definition 2. We call the multiplicative subgroup $\mathfrak{M}_{n}^{2}(N)$ of $M_{n}(\mathbb{C})$, an n-dimensional, order-2, $N$-generator (sub-)group (of $\left.M_{n}(\mathbb{C})\right)$.

Then, similar to the proof of the structure theorem Equation 14, one can obtain the following generalized result.

Theorem 5. Let $n \in \mathbb{N} \backslash\{1\}$ and $\mathfrak{M}_{n}^{2}(N)$ be an n-dimensional, order-2, $N$-generator group in $M_{n}(\mathbb{C})$. Then

$$
\mathfrak{M}_{n}^{2}(N) \stackrel{\text { Group }}{=} \Gamma_{N}^{2}
$$

Equation 44.

In particular, there exist generator-preserving group isomorphisms $\alpha^{n}: \Gamma_{N}^{2} \rightarrow \mathfrak{M}_{n}^{2}(N)$, such that

$$
\alpha^{n}\left(x_{j}\right)=X_{j} \text {, for all } j=1, \ldots, N,
$$

Equation 45.

where $x_{j}$ 's are the generators of $\Gamma_{N}^{2}$.

Proof. Let $n=2$. Then, by Equation 14, the isomorphic relation Equation 44 holds. Assume now that $n \geq 3$. Then the proof of Equation 44 is similar to that of Equation 14. Indeed, one can show that $\mathfrak{M}_{n}^{2}(N) \stackrel{\text { Group }}{=} \mathcal{F}_{N} / \mathcal{R}_{N} \stackrel{\text { Group }}{=} \Gamma_{N}^{2}$, for all $n \in \mathbb{N}$. Therefore, there exists a natural generator-preserving group-isomorphism $\alpha^{n}$ from $\Gamma_{N}^{2}$ onto $\mathfrak{M}_{n}^{2}(N)$ as in Equation 45.

This structure theorem is the generalized result of Equation 14. And the group-isomorphism $\alpha^{n}$ of Equation 45 generalizes $\alpha^{2}$ of Equation 17. We obtain the following theorem, generalizing Equation 3.

Theorem 6. Let $\Gamma_{N}^{2}$ be the group defined in Equation 1, for some $N \in \mathbb{N}$. Then there exists an n-dimensional Hilbert-space representation $\left(\mathbb{C}^{n}, \alpha^{n}\right)$ of $\Gamma_{N}^{2}$, where $\alpha^{n}$ are in the sense of Equation 45 , for all $n \in \mathbb{N} \backslash\{1\}$.

Proof. If $n=2$, as we have seen in Equation 3, there exists a 2-dimensional Hilbert-space representation $\left(\mathbb{C}^{2}, \alpha^{2}\right)$, where $\alpha^{2}$ is the group-isomorphism in the sense of Equation 17.

Suppose $n \geq 3$ in $\mathbb{N}$. For such $n$, two groups $\Gamma_{N}^{2}$ and $\mathfrak{M}_{n}^{2}(N)$ are isomorphic via a group-isomorphism $\alpha^{n}: \Gamma_{N}^{2} \rightarrow \mathfrak{M}_{n}^{2}(N)$ of Equation 45, by the previous theorem. It shows that the images $\alpha^{n}(g)$ are $(n \times n)$-matrices acting on the $n$-dimensional Hilbert space $\mathbb{C}^{n}$. So, the pair $\left(\mathbb{C}^{n}, \alpha^{n}\right)$ forms an $n$-dimensional representation of $\Gamma_{N}^{2}$, for all $n \in \mathbb{N}$.

The above two theorems show that, for the fixed group $\Gamma_{N}^{2}$, one has a system $\left\{\left(\mathbb{C}^{n}, \alpha^{n}\right)\right\}_{n=2}^{\infty}$ of Hilbert-space representations, and the corresponding isomorphic groups $\left\{\mathfrak{M}_{n}^{2}(N)\right\}_{n=2}^{\infty}$, acting on $\mathbb{C}^{n}$.

Example 1. Let $\Gamma_{2}^{2}$ be the finitely presented group, $\Gamma_{2}^{2}=\left\langle\left\{x_{1}, x_{2}\right\},\left\{x_{1}^{2}=x_{2}^{2}\right\}\right\rangle$. Fix $n=3$. Now, take the following strongly distinct triples, $W_{1}=\left(t_{1}, t_{2}, t_{3}\right), W_{2}=\left(s_{1}, s_{2}, s_{3}\right)$, in $\mathbb{C}_{\sim_{1}}^{\times} \times\left(\mathbb{C}^{\times}\right)^{2}$, and construct two matrices,

$$
A_{3}\left(W_{1}\right)=\left(\begin{array}{ccc}
1 & t_{3} & \frac{-t_{2} t_{3}}{1-t_{1}} \\
0 & t_{1} & t_{2} \\
0 & \frac{1-t_{1}^{2}}{t_{2}} & -t_{1}
\end{array}\right), A_{3}\left(W_{2}\right)=\left(\begin{array}{ccc}
1 & s_{3} & \frac{-s_{2} s_{3}}{1-s_{1}} \\
0 & s_{1} & s_{2} \\
0 & \frac{1-s_{1}^{2}}{s_{2}} & -s_{1}
\end{array}\right),
$$

Equation 46.

in $M_{3}(\mathbb{C})$. The group $\mathfrak{M}_{3}^{2}(2)$ is established as the reduced free group $\left\langle\left\{A_{3}\left(W_{1}\right), A_{3}\left(W_{2}\right)\right\}\right\rangle$ generated by $A_{3}\left(W_{1}\right)$ and $A_{3}\left(W_{2}\right)$ in $M_{3}(\mathbb{C})$. Then $\mathfrak{M}_{3}^{2}(2) \stackrel{\text { Group }}{=} \Gamma_{2}^{2}$.

So, one has a natural 3 -dimensional representation $\left(\mathbb{C}^{3}, \alpha^{3}\right)$, where $\alpha^{3}$ is the group-isomorphism satisfying $\alpha^{3}\left(x_{l}\right)=A_{3}\left(W_{l}\right)$, for all $l=1,2$. 


\section{APPLICATION: A GROUP ALGEBRA $\mathcal{A}_{2}$ INDUCED BY $\Gamma_{2}^{2}$}

In the previous section, we showed that the finitely presented group $\Gamma_{N}^{2}$ has a family of finite-dimensional Hilbert-space representations $\left\{\left(\mathbb{C}^{n}, \alpha^{n}\right)\right\}_{n=2}^{\infty}$, since it is group-isomorphic to $n$-dimensional, order-2, $N$-generator groups $\mathfrak{M}_{n}^{2}(N)$ in $M_{n}(\mathbb{C})$, for all $n \in \mathbb{N} \backslash\{1\}$, where $\mathfrak{M}_{n}^{2}(N)=\left\langle\left\{A_{n}\left(W_{k}\right)\right\}_{k=1}^{N}\right\rangle \in M_{n}(\mathbb{C})$, and where $W_{1}, \ldots, W_{N}$ are strongly mutually distinct $n$-tuples of $\mathbb{C}_{\sim 1}^{\times} \times\left(\mathbb{C}^{\times}\right)^{n-1}$, for $n \in \mathbb{N} \backslash\{1\}$.

\section{Algebraic Observation for Group Elements of $\Gamma_{2}^{2}$}

In this section, we concentrate on the case where $N=2$, studying the group elements of $\Gamma_{2}^{2}$ in detail. As a special case of Equation 44,

$$
\left\langle\left\{x_{1}, x_{2}\right\},\left\{x_{1}^{2}=x_{2}^{2}=e_{2}\right\}\right\rangle=\Gamma_{2}^{2} \stackrel{\text { Group }}{=} \mathfrak{M}_{2}^{2}(2) . \quad \text { Equation } 47 .
$$

Consider $\Gamma_{2}^{2}$ pure-algebraically. Each element $g$ of $\Gamma_{2}^{2}$ has its expression,

$$
g=x_{i_{1}}^{k_{1}} x_{i_{2}}^{k_{2}} \cdots x_{i_{n}}^{k_{i_{n}}}, \text { for some } n \in \mathbb{N},
$$

Equation 48 .

as in Equation 4, for some $\left(i_{1}, \ldots, i_{n}\right) \in\{1,2\}^{n}$, and $\left(k_{1}, \ldots, k_{n}\right) \in \mathbb{Z}^{n}$. However, by the relation on $\Gamma_{2}^{2}, x_{1}^{2}=e \stackrel{\text { denote }}{=}$ $e_{2}=x_{2}^{2} \in \Gamma_{2}^{2}$, one has that $x_{l}^{-1}=x_{l}$, for $l=1,2$, and $x_{l}^{2 k+1}=x_{l}$, for all $k \in \mathbb{N}$. In other words, the generators $x_{1}$ and $x_{2}$ are self-invertible, and indeed of order-2, in $\Gamma_{2}^{2}$. The above two conditions in can be summarized by

$$
x_{l}^{2 n+1}=x_{l}, \text { for } l=1,2 \text {, and for } n \in \mathbb{Z} .
$$

Equation 49.

So, the general expression of Equation 48 of $g$ is in fact

$$
g=x_{j_{1}} x_{j_{2}} \ldots x_{j_{n}} \in \Gamma_{2}^{2}
$$

Equation 50.

for some $\left(j_{1}, \ldots, j_{n}\right) \in\{1,2\}^{n}, n \in \mathbb{N}$, by Equation 49 .

By the characterization, $\Gamma_{2}^{2} \stackrel{\text { Group }}{=} \mathcal{F}_{2} / \mathcal{R}_{2}$, and by the definition of the noncommutative (non-reduced) free group $\mathcal{F}_{2}$, the expression Equation 50 of $g$ goes to

$$
g=\left\{\begin{array}{l}
e=e_{2} \\
x_{1} \\
x_{2} \\
\left(x_{1} x_{2}\right)^{n} x_{1} \\
\left(x_{1} x_{2}\right)^{n} \\
\left(x_{2} x_{1}\right)^{n} x_{2} \\
\left(x_{2} x_{1}\right)^{n}
\end{array}\right.
$$

Equation 51.

in $\Gamma_{2}^{2}$, for all $n \in \mathbb{N}$.

Proposition 7. Let $g \in \Gamma_{2}^{2}$. Then $g$ is only one of the forms in Equation 51.

Proof. Let $g \in \Gamma_{2}^{2} \backslash\left\{e, x_{1}, x_{2}\right\}$. Say, $g=x_{1} x_{2} x_{1} x_{2} \ldots x_{1} x_{2} x_{1}$. Then it is self-invertible. Indeed,

$$
\begin{aligned}
g^{2} & =\left(x_{1} x_{2} \ldots x_{1} x_{2} x_{1}\right)\left(x_{1} x_{2} \ldots x_{1} x_{2} x_{1}\right) \\
& =x_{1} x_{2} \ldots x_{1} x_{2}\left(x_{1}^{2}\right) x_{2} \ldots x_{1} x_{2} x_{1} \\
& =x_{1} x_{2} \ldots x_{1} x_{2} e x_{2} \ldots x_{1} x_{2} x_{1} \\
& =x_{1} x_{2} \ldots x_{1}\left(x_{2}^{2}\right) x_{1} \ldots x_{1} x_{2} x_{1} \\
& \vdots \\
& =x_{1}^{2} \\
& =e
\end{aligned}
$$

Equation 52. 
So

$$
\left(\left(x_{1} x_{2}\right)^{n} x_{1}\right)^{2}=e, \text { for all } n \in \mathbb{N}
$$

Equation 53.

Similarly, one obtains

$$
\left(\left(x_{2} x_{1}\right)^{n} x_{2}\right)^{2}=e, \text { for all } n \in \mathbb{N} .
$$

Equation 54.

Now, let $g=x_{1} x_{2} x_{1} x_{2} \ldots x_{1} x_{2} \in \Gamma_{2}^{2}$. Then $g^{-1}=x_{2} x_{1} x_{2} x_{1} \ldots x_{2} x_{1}$, with $|g|=\left|g^{-1}\right| \in \mathbb{N}$, where $|w|$ means the length of the word $w \in \Gamma_{2}^{2}$. Indeed, $g g^{-1}=\left(x_{1} x_{2} \ldots x_{1} x_{2}\right)\left(x_{2} x_{1} \ldots x_{2} x_{1}\right)=e$. So, one has that

$$
\left(\left(x_{1} x_{2}\right)^{n}\right)^{-1}=\left(x_{2} x_{1}\right)^{n},
$$

Equation 55.

and equivalently,

$$
\left(\left(x_{2} x_{1}\right)^{n}\right)^{-1}=\left(x_{1} x_{2}\right)^{n},
$$

Equation 56.

for all $n \in \mathbb{N}$.

Proposition 8. Let $g \in \Gamma_{2}^{2} \backslash\{e\}$, and let $|g|$ be the length of $g$ in $\left\{x_{1}, x_{2}\right\}$ in $\Gamma_{2}^{2}$.

1. If $|g|$ is odd then $g^{-1}=g$ in $\Gamma_{2}^{2}$.

2. If $|g|$ is even then $g=\left(x_{1} x_{2}\right)^{k}$, or $\left(x_{2} x_{1}\right)^{k}$, and $g^{-1}=\left(x_{2} x_{1}\right)^{k}$, respectively, $\left(x_{1} x_{2}\right)^{k}$, for some $k \in \mathbb{N}$.

Proof. Suppose $|g|$ is odd in $\mathbb{N}$. Then, by Equation 51, the group element $g$ is one of $x_{1}, x_{2},\left(x_{1} x_{2}\right)^{n} x_{1}$, or $\left(x_{2} x_{1}\right)^{n} x_{2}$, for $n \in \mathbb{N}$. By Equation 53 and Equation 54, in such cases, $g^{2}=e \in \Gamma_{2}^{2}$. So, the first statement holds.

Now, assume that $|g|$ is even in $\mathbb{N}$. Then, by Equation 51, this element $g$ is either $\left(x_{1} x_{2}\right)^{k}$, or $\left(x_{2} x_{1}\right)^{k}$, for $k \in \mathbb{N}$. By Equation 55 and Equation 56, respectively one has that

$$
\left(\left(x_{1} x_{2}\right)^{k}\right)^{-1}=\left(x_{2} x_{1}\right)^{k}, \text { and }\left(\left(x_{2} x_{1}\right)^{k}\right)^{-1}=\left(x_{1} x_{2}\right)^{k},
$$

Equation 57.

for all $k \in \mathbb{N}$. Therefore, the second statement holds, too.

Due to the self-invertibility of the group-identity, $e$, the generators $x_{1}, x_{2}$, and the group elements $g$ with odd length $|g|$, we are interested in the cases where $|g|$ is even: the cases where $g=\left(x_{1} x_{2}\right)^{k}$, or $g=\left(x_{2} x_{1}\right)^{k} \in \Gamma_{2}^{2}$.

Analytic and Combinatorial Observation for Elements of $\Gamma_{2}^{2}$ in $M_{2}(\mathbb{C})$

As we have seen, the group $\Gamma_{2}^{2}$ is group isomorphic to the 2-dimensional, order-2, 2-generator subgroup $\mathfrak{M}_{2}^{2}(2)$ of $M_{2}(\mathbb{C})$ under our representation $\left(\mathbb{C}^{2}, \alpha^{2}\right)$. In particular, $\mathfrak{M}_{2}^{2}(2)$ is generated by two matrices: $A_{2}\left(t_{1}, s_{1}\right)$ and $A_{2}\left(t_{2}, s_{2}\right)$ for the strongly mutually distinct pairs $\left(t_{1}, s_{1}\right)$ and $\left(t_{2}, s_{2}\right)$ in $\mathbb{C}^{\times} \times \mathbb{C}^{\times}$. More precisely,

$$
A_{2}\left(t_{1}, s_{1}\right)=\left(\begin{array}{cc}
t_{1} & s_{1} \\
\frac{1-t_{1}^{2}}{s_{1}} & -t_{1}
\end{array}\right) \text { and } A_{2}\left(t_{2}, s_{2}\right)=\left(\begin{array}{cc}
t_{2} & s_{2} \\
\frac{1-t_{2}^{2}}{s_{2}} & -t_{2}
\end{array}\right)
$$

Equation 58.

in $M_{2}(\mathbb{C})$.

In the rest of this paper, we denote $A_{2}\left(t_{1}, s_{1}\right)$ and $A_{2}\left(t_{2}, s_{2}\right)$, by $X_{1}$ and $X_{2}$, respectively, for convenience.

We are interested in group-elements $g$ formed by $g=\left(x_{1} x_{2}\right)^{k} \in \Gamma_{2}^{2}$, for $k \in \mathbb{N}$. In this case

$$
\alpha^{2}(g)=\alpha^{2}\left(\left(x_{1} x_{2}\right)^{k}\right)=\left(X_{1} X_{2}\right)^{k} \text {, and hence, }\left(\alpha^{2}(g)\right)^{-1}=\alpha^{2}\left(g^{-1}\right)=\left(X_{2} X_{1}\right)^{k},
$$

Equation 59.

in $\mathfrak{M}_{2}^{2}(2)$. 
Observe that

$$
\left(X_{1} X_{2}\right)+\left(X_{2} X_{1}\right)=\left(2 t_{1} t_{2}+\frac{s_{1}\left(1-t_{2}^{2}\right)}{s_{2}}+\frac{s_{2}\left(1-t_{1}^{2}\right)}{s_{1}}\right) I_{2}
$$

from the straightforward computation in $M_{2}(\mathbb{C})$.

Consider now that $\operatorname{det}\left(X_{1}\right)=-1=\operatorname{det}\left(X_{2}\right)$, and

$$
\begin{aligned}
\operatorname{det}\left(X_{1}+X_{2}\right) & =\operatorname{det}\left(\begin{array}{cc}
t_{1}+t_{2} & s_{1}+s_{2} \\
\frac{1-t_{1}^{2}}{s_{1}}+\frac{1-t_{2}^{2}}{s_{2}} & -t_{1}-t_{2}
\end{array}\right) \\
& =-\left(t_{1}^{2}+2 t_{1} t_{2}+t_{2}^{2}\right)-\left(1-t_{1}^{2}+\frac{s_{1}\left(1-t_{2}^{2}\right)}{s_{2}}+\frac{s_{2}\left(1-t_{1}^{2}\right)}{s_{1}}+1-t_{2}^{2}\right) \\
& =-t_{1}^{2}-2 t_{1} t_{2}-t_{2}^{2}-1+t_{1}^{2}-\frac{s_{1}\left(1-t_{2}^{2}\right)}{s_{2}}-\frac{s_{2}\left(1-t_{1}^{2}\right)}{s_{1}}-1+t_{2}^{2} \\
& =-2 t_{1} t_{2}-\frac{s_{1}\left(1-t_{2}^{2}\right)}{s_{2}}-\frac{s_{2}\left(1-t_{1}^{2}\right)}{s_{1}}-2
\end{aligned}
$$

Equation 61.

So, one can compute

$$
\operatorname{det}\left(X_{1}\right)+\operatorname{det}\left(X_{2}\right)-\operatorname{det}\left(X_{1}+X_{2}\right)=2 t_{1} t_{2}+\frac{s_{1}\left(1-t_{2}^{2}\right)}{s_{2}}+\frac{s_{2}\left(1-t_{1}^{2}\right)}{s_{1}} .
$$

Equation 62.

Lemma 9. Let $X_{1}$ and $X_{2}$ be the generating matrices of $\mathfrak{M}_{2}^{2}(2)$ as above. Then there exists $\varepsilon_{0} \in \mathbb{C}$, such that

$$
\begin{gathered}
\left(X_{1} X_{2}\right)+\left(X_{2} X_{1}\right)=\varepsilon_{0} I_{2} \in M_{2}(\mathbb{C}), \text { and } \\
\varepsilon_{0}=2 t_{1} t_{2}+\frac{s_{1}\left(1-t_{2}^{2}\right)}{s_{2}}+\frac{s_{2}\left(1-t_{1}^{2}\right)}{s_{1}} \\
=\operatorname{det}\left(X_{1}\right)+\operatorname{det}\left(X_{2}\right)-\operatorname{det}\left(X_{1}+X_{2}\right) .
\end{gathered}
$$

Equation 63.

Proof. The proof of Equation 63 is done by Equation 60 and Equation 62.

Therefore, one can observe that

$$
\begin{aligned}
\left(X_{1} X_{2}+X_{2} X_{1}\right)^{2} & =\left(X_{1} X_{2}\right)^{2}+X_{1} X_{2} X_{2} X_{1}+X_{2} X_{1} X_{1} X_{2}+\left(X_{2} X_{1}\right)^{2} \\
& =\left(X_{1} X_{2}\right)^{2}+\left(X_{2} X_{1}\right)^{2}+2 I_{2},
\end{aligned}
$$

and hence,

$$
\begin{aligned}
\left(X_{1} X_{2}\right)^{2}+\left(X_{2} X_{1}\right)^{2} & =\left(X_{1} X_{2}+X_{2} X_{1}\right)^{2}-2 I_{2} \\
& =\left(\varepsilon_{0} I_{2}\right)^{2}-2 I_{2} \\
& =\left(\varepsilon_{0}^{2}-2\right) I_{2} .
\end{aligned}
$$

By Equation 63 and Equation 65, we have that $\left(X_{1} X_{2}\right)^{1}+\left(X_{2} X_{1}\right)^{1}=\varepsilon_{0} I_{2}$, and $\left(X_{1} X_{2}\right)^{2}+\left(X_{2} X_{1}\right)^{2}=\left(\varepsilon_{0}^{2}-2\right) I_{2}$. More generally, we obtain the following recurrence relation. 
Theorem 10. Let $X_{1}$ and $X_{2}$ be the generating matrices of $\mathfrak{M}_{2}^{2}(2)$ in $M_{2}(\mathbb{C})$. If we denote $\mathfrak{X}_{k} \stackrel{\text { denote }}{=}\left(X_{1} X_{2}\right)^{k}+\left(X_{2} X_{1}\right)^{k}$, for all $k \in \mathbb{N}$, then the following recurrence relation is obtained.

$$
\begin{aligned}
& \mathfrak{X}_{1}=\varepsilon_{0} I_{2} \\
& \mathfrak{X}_{2}=\left(\varepsilon_{0}^{2}-2\right) I_{2} \\
& \quad \vdots \\
& \mathfrak{X}_{n}=\varepsilon_{0} \mathfrak{X}_{n-1}-\mathfrak{X}_{n-2}, \text { for all } n \geq 3 \in \mathbb{N},
\end{aligned}
$$

Equation 66.

in $M_{2}(\mathbb{C})$, where $\varepsilon_{0}=\operatorname{det}\left(X_{1}\right)+\operatorname{det}\left(X_{2}\right)-\operatorname{det}\left(X_{1}+X_{2}\right)$.

Proof. By Equation 63, indeed, one has $\mathfrak{X}_{1}=\varepsilon_{0} I_{2}$, and by Equation 65, $\mathfrak{X}_{2}=\left(\varepsilon_{0}^{2}-2\right) I_{2}$.

Suppose $n=3$ in $\mathbb{N}$. Then by Equation 51

$$
\begin{aligned}
\mathfrak{X}_{3} & =\left(X_{1} X_{2}\right)^{3}+\left(X_{2} X_{1}\right)^{3} \\
& =\left(X_{1} X_{2}+X_{2} X_{1}\right)^{3}-\left(3 X_{1} X_{2}+3 X_{2} X_{1}\right) \\
& =\left(\mathfrak{X}_{1}\right)^{3}-3 \mathfrak{X}_{1} \\
& =\left(\varepsilon_{0} I_{2}\right)^{3}-3\left(\varepsilon_{0} I_{2}\right) \\
& =\left(\varepsilon_{0}^{3}-3 \varepsilon_{0}\right) I_{2} .
\end{aligned}
$$

Equation 67.

Observe now that

$$
\begin{aligned}
\varepsilon_{0} \mathfrak{X}_{2}-\mathfrak{X}_{1} & =\varepsilon_{0}\left(\varepsilon_{0}^{2}-2\right) I_{2}-\varepsilon_{0} I_{2} \\
& =\left(\varepsilon_{0}^{3}-2 \varepsilon_{0}\right) I_{2}-\varepsilon_{0} I_{2} \\
& =\left(\varepsilon_{0}^{3}-3 \varepsilon_{0}\right) I_{2} .
\end{aligned}
$$

Equation 68.

Thus, one obtains that

$$
\begin{aligned}
\mathfrak{X}_{3} & =\left(\varepsilon_{0}^{3}-3 \varepsilon_{0}\right) \\
I_{2} & =\varepsilon_{0} \mathfrak{X}_{2}-\mathfrak{X}_{1} .
\end{aligned}
$$

Equation 69.

So, if $n=3$, then the relation in Equation 66 holds true.

Assume now that the statement

$$
\mathfrak{X}_{n_{0}}=\varepsilon_{0} \mathfrak{X}_{n_{0}-1}-\mathfrak{X}_{n_{0}-2}
$$

Equation 70.

holds for a fixed $n_{0} \geq 3$ in $\mathbb{N}$. Then

$$
\begin{aligned}
\mathfrak{X}_{n_{0}+1} & =\left(\left(X_{1} X_{2}\right)^{n_{0}}+\left(X_{2} X_{1}\right)^{n_{0}}\right)\left(X_{1} X_{2}+X_{2} X_{1}\right)-\left(X_{1} X_{2}\right)^{n_{0}}\left(X_{2} X_{1}\right)-\left(X_{2} X_{1}\right)^{n_{0}}\left(X_{1} X_{2}\right) \\
& =\left(\left(X_{1} X_{2}\right)^{n_{0}}+\left(X_{2} X_{1}\right)^{n_{0}}\right)\left(\varepsilon_{0} I\right)-\left(\left(X_{1} X_{2}\right)^{n_{0}-1}+\left(X_{2} X_{1}\right)^{n_{0}-1}\right) \\
& =\varepsilon_{0} \mathfrak{X}_{n_{0}}-\mathfrak{X}_{n_{0}-1},
\end{aligned}
$$

Equation 71.

Since $n_{0}$ is arbitrary in $\mathbb{N} \backslash\{1,2\}$, we can conclude that $\mathfrak{X}_{n+1}=\varepsilon_{0} \mathfrak{X}_{n}-\mathfrak{X}_{n-1}$, for all $n \in \mathbb{N} \backslash\{1\}$, by the induction.

Therefore, the relation in Equation 66 holds. 
By the recurrence relation Equation 66, we obtain that:

$$
\begin{aligned}
X_{1} X_{2}+X_{2} X_{1} & =\varepsilon_{0} I_{2}, \\
\left(X_{1} X_{2}\right)^{2}+\left(X_{2} X_{1}\right)^{2} & =\left(\varepsilon_{0}^{2}-2\right) I_{2}, \\
\left(X_{1} X_{2}\right)^{3}+\left(X_{2} X_{1}\right)^{3} & =\left(\varepsilon_{0}^{3}-3 \varepsilon_{0}\right) I_{2}, \\
\left(X_{1} X_{2}\right)^{4}+\left(X_{2} X_{1}\right)^{4} & =\varepsilon_{0}\left(\varepsilon_{0}^{3}-3 \varepsilon_{0}\right) I_{2}-\left(\varepsilon_{0}^{2}-2\right) I_{2} \\
& =\left(\varepsilon_{0}^{4}-4 \varepsilon_{0}^{2}+2\right) I_{2} .
\end{aligned}
$$

Equation 72.

Inductively, one can verify the following.

Corollary 11. There exists a functional sequence $\left(f_{n}\right)_{n=1}^{\infty}$, such that

$$
\left(X_{1} X_{2}\right)^{n}+\left(X_{2} X_{1}\right)^{n}=\left(f_{n}\left(\varepsilon_{0}\right)\right) I_{2} .
$$

Equation 73 .

The following theorem provides the refined result of Equation 73.

Theorem 12. Let $X_{1}$ and $X_{2}$ be the generating matrices of a 2-dimensional, order-2, 2-generator subgroup $\mathfrak{M}_{2}^{2}(2)$ of $M_{2}(\mathbb{C})$, and let $\mathfrak{X}_{l}=\left(X_{1} X_{2}\right)^{l}+\left(X_{2} X_{1}\right)^{l} \in M_{2}(\mathbb{C})$, for all $l \in \mathbb{N}$. Then there exists a functional sequence $\left(f_{n}\right)_{n=1}^{\infty}$ such that $\mathfrak{X}_{n}=$ $f_{n}\left(\varepsilon_{0}\right) I_{2} \in M_{2}(\mathbb{C})$, where $\varepsilon_{0}=\operatorname{det}\left(X_{1}\right)+\operatorname{det}\left(X_{2}\right)-\operatorname{det}\left(X_{1}+X_{2}\right)$. Moreover,

$$
\begin{aligned}
f_{n}(z) & =\sum_{k=0}^{\left[\frac{n}{2}\right]}(-1)^{k} \frac{n}{k}\left(\begin{array}{c}
n-k-1 \\
k-1
\end{array}\right) z^{n-2 k} \\
& =\sum_{k=0}^{\left[\frac{n}{2}\right]}(-1)^{k} \frac{n}{n-k}\left(\begin{array}{c}
n-k \\
k
\end{array}\right) z^{n-2 k}
\end{aligned}
$$

Equation 74.

where $\left[\frac{n}{2}\right]$ is the maximal integer less than or equal to $\frac{n}{2}$.

Proof. By Equation 66 and Equation 73, there exists a functional sequence $\left(f_{n}\right)_{n=1}^{\infty}$ such that $\mathfrak{X}_{n}=f_{n}\left(\varepsilon_{0}\right) I_{2}$, for all $n \in \mathbb{N}$. For instance,

$$
\begin{aligned}
& f_{1}(z)=z \\
& f_{2}(z)=z^{2}-2 \\
& f_{3}(z)=z^{3}-3 z \\
& f_{4}(z)=z^{4}-4 z^{2}+2
\end{aligned}
$$

Equation 75.

So, it suffices to show that each $n$-th entry of the sequence $f_{n}(z)$ satisfies Equation 74 , for all $n \in \mathbb{N}$.

Say $n=1$. The function $f_{1}(z)$ satisfies

$$
\begin{aligned}
f_{1}(z) & =\sum_{k=0}^{\left[\frac{1}{2}\right]}(-1)^{k} \frac{n}{n-k}\left(\begin{array}{c}
n-k-1 \\
k-1
\end{array}\right) z^{n-2 k} \\
& =\sum_{k=0}^{0}(-1)^{k} \frac{n}{n-k}\left(\begin{array}{c}
n-k \\
k
\end{array}\right) z^{n-2 k} \\
& =(-1)^{0} \frac{1}{1-0}\left(\begin{array}{c}
1-0 \\
0
\end{array}\right) z^{1-0} \\
& =z
\end{aligned}
$$

Equation 76. 
If $n=2$, then the function $f_{2}(z)$ is

$$
\begin{aligned}
f_{2}(z) & =\sum_{k=0}^{1}(-1)^{k} \frac{n}{n-k}\left(\begin{array}{c}
n-k \\
k
\end{array}\right) z^{n-2 k} \\
& =(-1)^{0} \frac{2}{2-0}\left(\begin{array}{c}
2-0 \\
0
\end{array}\right) z^{2-0}+(-1)^{1} \frac{2}{2-1}\left(\begin{array}{c}
2-1 \\
1
\end{array}\right) z^{2-2} \\
& =z^{2}-1
\end{aligned}
$$

Equation 77.

Therefore, the formula Equation 74 holds true where $n=1,2$, by Equation 75 .

Now, we consider the recurrence relation.

$$
\begin{aligned}
f_{1}(z) & =z \\
f_{2}(z) & =z^{2}-1 \\
f_{n+1}(z) & =z f_{n}(z)-f_{n-1}(z)
\end{aligned}
$$

Equation 78 .

for all $n \geq 2$ in $\mathbb{N}$.

First of all, the functions $f_{1}$ and $f_{2}$ satisfy the initial condition of the relation by Equation 76 and Equation 77 . So, concentrate on the cases where $n \geq 2$. Observe that

$$
\begin{aligned}
& z f_{n}(z)-f_{n-1}(z)=z\left(\sum_{k=0}^{\left[\frac{n}{2}\right]}(-1)^{k} \frac{n}{k}\left(\begin{array}{c}
n-k-1 \\
k-1
\end{array}\right) z^{n-2 k}\right)-\sum_{k=0}^{\left[\frac{n-1}{2}\right]}(-1)^{k} \frac{n-1}{k}\left(\begin{array}{c}
n-k-2 \\
k-1
\end{array}\right) z^{n-1-2 k} \\
& =\sum_{k=0}^{\left[\frac{n}{2}\right]}(-1)^{k} \frac{n}{k}\left(\begin{array}{c}
n-k-1 \\
k-1
\end{array}\right) z^{n-2 k+1}-\sum_{k=1}^{\left[\frac{n+1}{2}\right]}(-1)^{k-1} \frac{n-1}{k-1}\left(\begin{array}{c}
n-k-1 \\
k-2
\end{array}\right) z^{n-1-2 k+2} \\
& =\sum_{k=0}^{\left[\frac{n}{2}\right]}(-1)^{k} \frac{n}{k}\left(\begin{array}{c}
n-k-1 \\
k-1
\end{array}\right) z^{n+1-2 k}+\sum_{k=1}^{\left[\frac{n+1}{2}\right]}(-1)^{k} \frac{n-1}{k-1}\left(\begin{array}{c}
n-k-1 \\
k-2
\end{array}\right) z^{n+1-2 k} \\
& =z^{n+1}+\sum_{k=1}^{\left[\frac{n}{2}\right]}(-1)^{k} \frac{n}{k}\left(\begin{array}{c}
n-k-1 \\
k-1
\end{array}\right) z^{n+1-2 k}+\sum_{k=1}^{\left[\frac{n+1}{2}\right]}(-1)^{k} \frac{n-1}{k-1}\left(\begin{array}{c}
n-k-1 \\
k-2
\end{array}\right) z^{n+1-2 k} \\
& =z^{n+1}+\sum_{k=1}^{\left[\frac{n}{2}\right]}(-1)^{k}\left(\frac{n}{k}\left(\begin{array}{c}
n-k-1 \\
k-1
\end{array}\right)+\frac{n-1}{k-1}\left(\begin{array}{c}
n-k-1 \\
k-2
\end{array}\right)\right) z^{n+1-2 k} \\
& +(-1)^{\left[\frac{n+1}{2}\right]} \frac{n-1}{\left[\frac{n+1}{2}\right]-1}\left(\begin{array}{c}
n-\left[\frac{n+1}{2}\right]-1 \\
{\left[\frac{n+1}{2}\right]-1}
\end{array}\right) z^{n+1-2\left[\frac{n+1}{2}\right]} \\
& =z^{n+1}+\sum_{k=1}^{\left[\frac{n}{2}\right]}(-1)^{k}\left(\frac{n(n-k-1) !}{k(n-k-1-k+1) !(k-1) !}+\frac{(n-1)(n-k-1) !}{(k-1)(n-k+k+2) !(k-2) !}\right) z^{n+1-2 k} \\
& +\mathfrak{Y} \\
& \text { Equation } 79 .
\end{aligned}
$$


where

$$
\begin{aligned}
& \mathfrak{Y}=(-1)^{\left[\frac{n+1}{2}\right]} \frac{n-1}{\left[\frac{n+1}{2}\right]-1}\left(\begin{array}{c}
n-\left[\frac{n+1}{2}\right]-1 \\
{\left[\frac{n+1}{2}\right]-1}
\end{array}\right) z^{n+1-2\left[\frac{n+1}{2}\right]}, \\
&=z^{n+1}+\sum_{k=1}^{\left[\frac{n}{2}\right]}(-1)^{k}\left(\frac{n(n-k-1) !}{(n-2 k) ! k !}+\frac{n(n-k-1) !-(n-k-1) !}{(n-2 k+1) !(k-1) !}\right) z^{n+1-2 k}+\mathfrak{Y} \\
&=z^{n+1}+\sum_{k=1}^{\left[\frac{n}{2}\right]}(-1)^{k}\left(\frac{(n-2 k+1) n(n-k-1) !+k n(n-k-1) !-k(n-k-1) !}{k(n-2 k+1) !(k-1) !}\right) z^{n+1-2 k}+\mathfrak{Y} \\
&=z^{n+1}+\sum_{k=1}^{\left[\frac{n}{2}\right]}(-1)^{k}\left(\frac{\left(n^{2}-2 k n+n+n k-k\right)(n-k-1) !}{k(n-2 k+1) !(k-1) !}\right) z^{n+1-2 k}+\mathfrak{Y} \\
&=z^{n+1}+\sum_{k=1}^{\left[\frac{n}{2}\right]}(-1)^{k}\left(\frac{\left(n^{2}-n k+n-k\right)(n-k-1) !}{k(n-2 k+1) !(k-1) !}\right) z^{n+1-2 k}+\mathfrak{Y} \\
&=z^{n+1}+\sum_{k=1}^{\left[\frac{n}{2}\right]}(-1)^{k}\left(\frac{(n+1)(n-k)(n-k-1) !}{k(n-2 k+1) !(k-1) !}\right) z^{n+1-2 k}+\mathfrak{Y} \\
&=z^{n+1}+\sum_{k=1}^{\left[\frac{n}{2}\right]}(-1)^{k} \frac{n+1}{k} \frac{(n-k) !}{(n-k-(k-1)) !(k-1) !} z^{n+1-2 k}+\mathfrak{Y} \\
&=z^{n+1}+\sum_{k=1}^{\left[\frac{n}{2}\right]}(-1)^{k} \frac{n+1}{k}\left(\begin{array}{c}
n-k \\
k-1
\end{array}\right) z^{n+1-2 k}+\mathfrak{Y} \\
&=\sum_{k=0}^{\left[\frac{n}{2}\right]}(-1)^{k} \frac{n+1}{k}\left(\begin{array}{c}
n-k \\
k-1
\end{array}\right) z^{n+1-2 k}+\mathfrak{Y} . \\
& E q
\end{aligned}
$$

Equation 80 .

For any $n \geq 2$, we obtain

$$
z f_{n}(z)-f_{n-1}(z)=\sum_{k=0}^{\left[\frac{n}{2}\right]}(-1)^{k} \frac{n+1}{k}\left(\begin{array}{c}
n-k \\
k-1
\end{array}\right) z^{n+1-2 k}+\mathfrak{Y} .
$$

Equation 81.

Notice that if $n \geq 2$ is even in $\mathbb{N}$, then $\left[\frac{n}{2}\right]=\left[\frac{n+1}{2}\right]$ in $\mathbb{N}$ and hence, the first term of Equation 81 contains the second one. Therefore, one has

$$
n \in 2 \mathbb{N} \Rightarrow z f_{n}(z)-f_{n-1}(z)=\sum_{k=0}^{\left[\frac{n+1}{2}\right]}(-1)^{k} \frac{n+1}{k}\left(\begin{array}{c}
n-k \\
k-1
\end{array}\right) z^{n+1-2 k},
$$

if and only if $z f_{n}(z)-f_{n-1}(z)=f_{n+1}(z)$ for all $n \in 2 \mathbb{N}$. 
Now, assume $n \geq 2$ is odd. Then

$$
\begin{aligned}
& \mathfrak{Y}=(-1)^{\left[\frac{n+1}{2}\right]}\left(\frac{2 n-2}{n+1-2}\right)\left(\frac{\left(n-\left[\frac{n+1}{2}\right]-1\right) !}{\left(n-\left[\frac{n+1}{2}\right]-1-\left[\frac{n+1}{2}\right]+2\right) !\left(\left[\frac{n+1}{2}\right]-2\right) !}\right) z^{n+1-2\left[\frac{n+1}{2}\right]} \\
&=(-1)^{\left[\frac{n+1}{2}\right]}\left(\frac{2 n-2}{n-1}\right)\left(\frac{\left(n-\left[\frac{n+1}{2}\right]-1\right) !}{\left(n-2\left[\frac{n+1}{2}\right]+1\right) !\left(\left[\frac{n+1}{2}\right]-2\right) !}\right) z^{0} \\
&=(-1)^{\left[\frac{n+1}{2}\right]}(2)\left(\frac{\left(n-\left[\frac{n+1}{2}\right]\right) !}{\left(n-2\left[\frac{n+1}{2}\right]+1\right) !\left(n-\left[\frac{n+1}{2}\right]\right)\left(\left[\frac{n+1}{2}\right]-2\right) !}\right) z^{0} \\
&=(-1)^{\left[\frac{n+1}{2}\right]}\left(\frac{n+1}{\left[\frac{n+1}{2}\right]}\right)\left(\frac{\left[\frac{n+1}{2}\right]-1}{n-\left[\frac{n+1}{2}\right]}\right)\left(\begin{array}{c}
n-\left[\frac{n+1}{2}\right] \\
{\left[\frac{n+1}{2}\right]-1}
\end{array}\right) z^{0} \\
&=(-1)^{\left[\frac{n+1}{2}\right]}\left(\frac{n+1}{\left[\frac{n+1}{2}\right]}\right)\left(\frac{n+1-2}{2 n-n-1}\right)\left(\begin{array}{c}
n-\left[\frac{n+1}{2}\right] \\
{\left[\frac{n+1}{2}\right]-1}
\end{array}\right) z^{0} \\
&=\left(\begin{array}{c}
n-\left[\frac{n+1}{2}\right] \\
{\left[\frac{n+1}{2}\right]}
\end{array}\right) z^{0} . \\
& {\left[\frac{n+1}{\left[\frac{n+1}{2}\right]}\right)\left(\begin{array}{c}
{\left[\frac{n+1}{2}\right]-1}
\end{array}\right) }
\end{aligned}
$$

Therefore

$$
n \in 2 \mathbb{N}+1 \Longrightarrow \mathfrak{Y}=\left((-1)^{\left[\frac{n+1}{2}\right]}\left(\frac{n+1}{\left[\frac{n+1}{2}\right]}\right)\left(\begin{array}{c}
n-\left[\frac{n+1}{2}\right] \\
{\left[\frac{n+1}{2}\right]-1}
\end{array}\right)\right) z^{0}
$$

Equation 84.

So, if $n \geq 2$ is odd then

$$
z f_{n}(z)-f_{n-1}(z)=\sum_{k=0}^{\left[\frac{n+1}{2}\right]}(-1)^{k} \frac{n+1}{k}\left(\begin{array}{c}
n-k \\
k-1
\end{array}\right) z^{n+1-2 k},
$$

if and only if $z f_{n}(z)-f_{n-1}(z)=f_{n+1}(z)$. Therefore, by Equation 82 and Equation 85, one can conclude that

$$
n \geq 2 \in \mathbb{N} \rightarrow z f_{n}(z)-f_{n-1}(z)=f_{n+1}(z) .
$$

Equation 86.

The above result shows that $f_{n+1}\left(\varepsilon_{0}\right)=\varepsilon_{0} f_{n}\left(\varepsilon_{0}\right)-f_{n-1}\left(\varepsilon_{0}\right)$, for $n \geq 2 \in \mathbb{N}$, with $f_{1}\left(\varepsilon_{0}\right)=\varepsilon_{0}$, and $f_{2}\left(\varepsilon_{0}\right)=\varepsilon_{0}^{2}-2$. By Equation 66, one can conclude that if

$$
f_{n}(z)=\sum_{k=0}^{\left[\frac{n}{2}\right]}(-1)^{k}\left(\frac{n}{k}\right)\left(\begin{array}{c}
n-k-1 \\
k-1
\end{array}\right) z^{n-2 k} \text {, then } \mathfrak{X}_{n}=f_{n}\left(\varepsilon_{0}\right) I_{2}
$$

Equation 87.

for all $n \in \mathbb{N}$.

This result shows that the generators $x_{1}$ and $x_{2}$ of $\Gamma_{2}^{2}$ induces the analytic data in $M_{2}(\mathbb{C})$ depending on the elements $x_{1} x_{2}$ and their inverses $x_{2} x_{1}$ up to Equation 87.

Group Algebras $A_{\alpha^{n}, \Gamma_{N}^{2}}$

Let $\Gamma$ be an arbitrary discrete group, and let $(H, \alpha)$ be a Hilbert-space representation of $\Gamma$ consisting of a Hilbert-space $H$ and the group-action $\alpha: \Gamma \rightarrow L(H)$, making $\alpha(g): H \rightarrow H$, for all $g \in \Gamma$ be linear operators (or linear transformations) on $H$, satisfying $\alpha\left(g_{1} g_{2}\right)=\alpha\left(g_{1}\right) \alpha\left(g_{2}\right)$, for all $g_{1}, g_{2} \in \Gamma$, and $\alpha\left(g^{-1}\right)=(\alpha(g))^{-1}$, for all $g \in \Gamma$, where $L(H)$ is the operator algebra consisting of all linear operators on $H$. 
Note that, if we are working with topologies, then one may replace $L(H)$ with $B(H)$, the operator algebra consisting of all bounded (or continuous) linear operators on $H$. However, we are not considering topologies throughout this paper, so we simply set the representational models for $L(H)$ here.

Our group $\Gamma_{N}^{2}$ has its Hilbert-space representations $\left(\mathbb{C}^{n}, \alpha^{n}\right)$, for all $n \in \mathbb{N} \backslash\{1\}$, and each element $g$ of $\Gamma_{N}^{2}$ is realized as $\alpha^{n}(g) \in \mathfrak{M}_{n}^{2}(N)$ in

$$
M_{n}(\mathbb{C})=L\left(\mathbb{C}^{n}\right)=B\left(\mathbb{C}^{n}\right) . \quad \text { Equation } 88 .
$$

The first equality holds because, under finite-dimensionality, all linear operators on $\mathbb{C}^{n}$ are matrices acting on $\mathbb{C}^{n}$, and vice versa. The second equality holds, because, under finite-dimensionality, all pure-algebraic operators on $\mathbb{C}^{n}$ are automatically bounded (and hence, continuous).

For an arbitrary group $\Gamma$, realized by $(H, \alpha)$, one obtains the isomorphic group $\alpha(\Gamma)$ in $L(H)$, i.e., $\Gamma \stackrel{\text { Group }}{=} \alpha(\Gamma) \in L(H)$. So, one can construct a subalgebra,

$$
A_{\alpha, \Gamma}=\mathbb{C}[\alpha(\Gamma)] \text { of } L(H)
$$

Equation 89.

where $\mathbb{C}[X]$ is the polynomial ring over the set $X$. Such rings $\mathbb{C}[X]$ form algebras under polynomial addition and polynomial multiplication over $\mathbb{C}$, and hence, under the inherited operator addition and operator multiplication, $\mathbb{C}[\alpha(\Gamma)]$ forms an algebra in $L(H)$.

Definition 3. Let $\Gamma$ be a group and let $(H, \alpha)$ be a Hilbert-space representation of $\Gamma$. The subalgebra $A_{\alpha, \Gamma}$ of $L(H)$ in the sense of Equation 89 is called the group algebra of $\Gamma$ induced by $(H, \alpha)$.

It is not difficult to show that if

$$
A_{\Gamma}^{o}=\mathbb{C}[\Gamma]
$$

Equation 90.

the pure-algebraic algebra generated by $\Gamma$, as a polynomial ring in $\Gamma$, then

$$
A_{\Gamma}^{o} \stackrel{\mathrm{Alg}}{=} A_{\alpha, \Gamma},
$$

Equation 91.

for all Hilbert-space representations $(H, \alpha)$ of $\Gamma$ algebraically, where “Alg”" means “algebra isomorphic to.”

Proposition 13. Let $\Gamma_{N}^{2}$ be the group defined in Equation 1, and let $\left(\mathbb{C}^{n}, \alpha^{n}\right)$ be our n-dimensional representations of $\Gamma_{N}^{2}$, for all $n \in \mathbb{N} \backslash\{1\}$. Let $\mathfrak{M}_{n}^{2}(N)$ be the $n$-dimensional, order-2, $N$-generator subgroups of $M_{n}(\mathbb{C})$, for all $n \in \mathbb{N} \backslash\{1\}$. If $A_{\alpha^{n}, \Gamma_{N}^{2}}$ are in the sense of Equation 89, and $A_{\Gamma_{N}^{2}}$ is the group algebra in the sense of Equation 90 , then

$$
A_{\Gamma_{N}^{2}} \stackrel{\text { Alg }}{=} A_{\alpha^{n}, \Gamma_{N}^{2}}=\mathbb{C}\left[\mathfrak{M}_{n}^{2}(N)\right], \text { for all } n \in \mathbb{N} .
$$

Equation 92.

Proof. The proof is deduced from Equation 91. Recall that $\alpha^{n}\left(\Gamma_{2}^{2}\right)=\mathfrak{M}_{n}^{2}(N)$, for all $n \in \mathbb{N}$.

A Group Algebra $A_{\alpha^{2}}, \Gamma_{2}^{2}$

In this section, we take the group algebra in the sense of Equation 89,

$$
A_{\alpha^{2}, \Gamma_{2}^{2}} \stackrel{\text { denote }}{=} \mathcal{A}_{2}
$$

Equation 93.

as a subalgebra of $M_{2}(\mathbb{C})=L\left(\mathbb{C}^{2}\right)$, under our representation $\left(\mathbb{C}^{2}, \alpha^{2}\right)$. By Equation 92, pure-algebraically, the algebra $\mathcal{A}_{2}$ of is algebra isomorphic to the group algebra $A_{\Gamma_{2}^{2}}=\mathbb{C}\left[\Gamma_{2}^{2}\right]$.

By Equation 51, all elements of $\mathcal{A}_{2}$ are the linear combinations of the matrices formed by

$$
I_{2}, X_{1}, X_{2},\left(X_{1} X_{2}\right)^{n},\left(X_{2} X_{1}\right)^{n} \text {, or }\left(X_{1} X_{2}\right)^{n} X_{1},\left(X_{2} X_{1}\right)^{n} X_{2},
$$

Equation 94. 
for all $n \in \mathbb{N}$, where $X_{j}=\alpha^{2}\left(x_{j}\right)$, for $j=1,2$, since $\mathcal{A}_{2}=\mathbb{C}\left[\mathfrak{M}_{2}^{2}(2)\right] \in M_{2}(\mathbb{C})$.

As we have seen in the previous section, the building blocks of $\mathcal{A}_{2}$ satisfy the following.

(i) $I_{2}, X_{1}, X_{2},\left(X_{1} X_{2}\right)^{n} X_{1}$, and $\left(X_{2} X_{1}\right)^{n} X_{2}$ are self-invertible in $\mathcal{A}_{2} \subset M_{2}(\mathbb{C})$.

(ii) $\left(\left(X_{1} X_{2}\right)^{n}\right)^{-1}=\left(X_{2} X_{1}\right)^{n}$, for all $n \in \mathbb{N}$.

Corollary 14. Let $X_{1}$ and $X_{2}$ be the generating matrices of $\mathfrak{M}_{2}^{2}(2)$, and hence, those of $\mathcal{A}_{2}$. Then

$$
\begin{aligned}
& \left(\left(X_{1} X_{2}\right)^{n}\right)^{-1}=f_{n}\left(\varepsilon_{0}\right) I_{2}-\left(X_{1} X_{2}\right)^{n} \\
& \left(\left(X_{2} X_{1}\right)^{n}\right)^{-1}=f_{n}\left(\varepsilon_{0}\right) I_{2}-\left(X_{2} X_{1}\right)^{n}
\end{aligned}
$$

Equation 95.

for all $n \in \mathbb{N}$, where $\left(f_{n}\right)_{n=2}^{\infty}$ is the functional sequence from Equation 74 .

Proof. By Equation 87, we have $\left(X_{1} X_{2}\right)^{n}+\left(X_{2} X_{1}\right)^{n}=f_{n}\left(\varepsilon_{0}\right) I_{2} \in \mathcal{A}_{2}$, where $f_{n}$ are in the sense of Equation 74, and $\varepsilon_{0}$ is in the sense of Equation 63, for all $n \in \mathbb{N}$. So,

$$
\left(X_{2} X_{1}\right)^{n}=f_{n}\left(\varepsilon_{0}\right) I_{2}-\left(X_{1} X_{2}\right)^{n} \in \mathcal{A}_{2}
$$

Equation 96.

for all $n \in \mathbb{N}$. Thus,

$$
\left(\left(X_{1} X_{2}\right)^{n}\right)^{-1}=f_{n}\left(\varepsilon_{0}\right) I_{2}-\left(X_{1} X_{2}\right)^{n}
$$

Equation 97.

for all $n \in \mathbb{N}$.

Similarly, we obtain $\left(\left(X_{2} X_{1}\right)^{n}\right)^{-1}=f_{n}\left(\varepsilon_{0}\right) I_{2}-\left(X_{2} X_{1}\right)^{n}$, for all $n \in \mathbb{N}$.

It means that if $T$ is a matrix of $\mathcal{A}_{2}$ in the form of either $\left(X_{1} X_{2}\right)^{n}$ or $\left(X_{2} X_{1}\right)^{n}$ for any $n \in \mathbb{N}$, then $T^{-1}=f_{n}\left(\varepsilon_{0}\right) I_{2}-T$.

Proposition 15. Let $A \in \mathfrak{M}_{2}^{2}(2)$ in $\mathcal{A}_{2}$. Then

$$
A^{-1}=\left\{\begin{array}{l}
A \\
r_{A} I_{2}-A
\end{array}\right.
$$

Equation 98.

for some $r_{A} \in \mathbb{C}$.

Proof. If $A \in \mathfrak{M}_{2}^{2}(2)$ in $\mathcal{A}_{2}$, then $T$ is one of the forms listed in Equation 94. As we discussed above, if $A$ is $I_{2}, X_{1}, X_{2}$, $\left(X_{1} X_{2}\right)^{n} X_{1}$, or $\left(X_{2} X_{1}\right)^{n} X_{2}$, for $n \in \mathbb{N}$, then it is self-invertible in $\mathcal{A}_{2}$. i.e., $A^{-1}=A$ in $\mathcal{A}_{2}$.

If $A$ is either $\left(X_{1} X_{2}\right)^{n}$, or $\left(X_{2} X_{1}\right)^{n}$, for any $n \in \mathbb{N}$, then, by Equation 95, there exists $r_{A} \in \mathbb{C}$, such that $A^{-1}=r_{A}-A$ in $\mathcal{A}_{2}$.

The above proposition characterizes the invertibility of $\mathfrak{M}_{2}^{2}(2)$ in $\mathcal{A}_{2}$.

Trace of Certain Matrices of $\mathcal{A}_{2}$

Let $\mathcal{A}_{2}$ be the group algebra of $\Gamma_{2}^{2}$ induced by $\left(\mathbb{C}^{2}, \alpha^{2}\right)$ in $M_{2}(\mathbb{C})$, in the sense of Equation 93. Since the algebra $\mathcal{A}_{2}$ is a subalgebra of $M_{2}(\mathbb{C})$, one can naturally restrict the trace $\operatorname{tr}$ on $M_{2}(\mathbb{C})$ to that on $\mathcal{A}_{2}$. i.e., the pair $\left(\mathcal{A}_{2}, t r\right)$ forms a noncommutative free probability space. ${ }^{7,8}$

In this section, we are interested in trace of certain types of matrices in $\mathcal{A}_{2}$. Let $X_{1}$ and $X_{2}$ be the generating matrices of $\mathfrak{M}_{2}^{2}(2)$, and hence, those of $\mathcal{A}_{2}$. Define a new element $T$ of $\mathcal{A}_{2}$ by

$$
T=X_{1}+X_{2} \in \mathcal{A}_{2} \text {. }
$$

Equation 99. 
By the self-invertibility of $X_{1}$ and $X_{2}$, this matrix is understood as the radial operator of $\mathcal{A}_{2} \cdot{ }^{5}$

Observe that

$$
\begin{aligned}
T^{2} & =\left(X_{1}+X_{2}\right)^{2} \\
& =X_{1}^{2}+\left(X_{1} X_{2}+X_{2} X_{1}\right)+X_{2}^{2} \\
& =I_{2}+f_{1}\left(\varepsilon_{0}\right) I_{2}+I_{2} \\
& =\left(f_{1}\left(\varepsilon_{0}\right)+2\right) I_{2} \\
T^{3} & =\left(X_{1}+X_{2}\right)^{3} \\
& =\left(X_{1}+X_{2}\right)^{2}\left(X_{1}+X_{2}\right) \\
& =\left(f_{1}\left(\varepsilon_{0}\right)+2\right) T \\
T^{4} & =T^{3} T \\
& =\left(f_{1}\left(\varepsilon_{0}\right)+2\right) T^{2} \\
& =\left(f_{1}\left(\varepsilon_{0}\right)+2\right)^{2} I_{2} \\
T^{5} & =T^{4} T \\
& =\left(f_{1}\left(\varepsilon_{0}\right)+2\right)^{2} T \\
T^{6} & =T^{5} T \\
& =\left(f_{1}\left(\varepsilon_{0}\right)+2\right)^{2} T^{2} \\
& =\left(f_{1}\left(\varepsilon_{0}\right)+2\right)^{3} I_{2}
\end{aligned}
$$

where $f_{n}$ are in the sense of Equation 74,

$$
f_{n}(z)=\sum_{k=0}^{\left[\frac{n}{2}\right]}(-1)^{k}\left(\frac{n}{k}\right)\left(\begin{array}{c}
n-k-1 \\
k-1
\end{array}\right) z^{n-2 k},
$$

Equation 101.

for all $n \in \mathbb{N}$, and hence, $f_{1}\left(\varepsilon_{0}\right)=\varepsilon_{0}$. Inductively, one obtains the following.

Theorem 16. Let $T=X_{1}+X_{2}$ be the radial operator of $\mathcal{A}_{2}$, where $X_{1}$ and $X_{2}$ are the generating matrices of $\mathcal{A}_{2}$. Then

$$
\begin{aligned}
T^{2 n} & =\left(\varepsilon_{0}+2\right)^{n} I_{2} \\
T^{2 n+1} & =\left(\varepsilon_{0}+2\right)^{n} T,
\end{aligned}
$$

in $\mathcal{A}_{2}$, for all $n \in \mathbb{N}$, where $\varepsilon_{0}=\operatorname{det}\left(X_{1}\right)+\operatorname{det}\left(X_{2}\right)-\operatorname{det}\left(X_{1}+X_{2}\right)$.

Proof. The proof is inductive and relies on the observations above. Indeed, one can find $T^{2 n}=\left(f_{1}\left(\varepsilon_{0}\right)+2\right)^{n} I_{2}$ and $T^{2 n+1}=\left(f_{1}\left(\varepsilon_{0}\right)+2\right)^{n} T$ in $\mathcal{A}_{2}$ for all $n \in \mathbb{N}$. Finally, since $f_{1}(z)=z$, we obtain $f_{1}\left(\varepsilon_{0}\right)=\varepsilon_{0}$.

Corollary 17. Let $T=n X_{1}+X_{2}$ be the radial operator of $\mathcal{A}_{2}$. Then

$$
\operatorname{tr}\left(T^{n}\right)= \begin{cases}2\left(\varepsilon_{0}+2\right)^{n / 2} & \text { if } n \text { is even } \\ 0 & \text { if } n \text { is odd }\end{cases}
$$

Equation 103.

for $n \in \mathbb{N}$. 
Proof. Set

$$
X_{j}=\left(\begin{array}{cc}
t_{j} & s_{j} \\
\frac{1-t_{j}^{2}}{s_{j}} & -t_{j}
\end{array}\right) \in \mathcal{A}_{2}, \text { for } j=1,2
$$

Equation 104.

where $\left(t_{1}, s_{1}\right)$ and $\left(t_{2}, s_{2}\right)$ are strongly distinct pair in $\mathbb{C}^{\times} \times \mathbb{C}^{\times}$. By Equation 102, if $T$ is given as above in $\mathcal{A}_{2}$, then $T^{2 n}=\left(\varepsilon_{0}+2\right)^{n} I_{2}$, and $T^{2 n+1}=\left(\varepsilon_{0}+2\right)^{n} T$, for $n \in \mathbb{N}$. Thus, it is not difficult to check that

$$
\begin{aligned}
\operatorname{tr}\left(T^{2 n}\right) & =\operatorname{tr}\left(\left(\begin{array}{cc}
\left(\varepsilon_{0}+2\right)^{n} & 0 \\
0 & \left(\varepsilon_{0}+2\right)^{n}
\end{array}\right)\right) \\
& =\left(\varepsilon_{0}+2\right)^{n}+\left(\varepsilon_{0}+2\right)^{n} \\
& =2\left(\varepsilon_{0}+2\right)^{n},
\end{aligned}
$$

Equation 105.

for any $n \in \mathbb{N}$, By the direct computation and by Equation 104, one has

$$
T=\left(\begin{array}{cc}
t_{1}+t_{2} & s_{1}+s_{2} \\
\frac{1-t_{1}^{2}}{s_{1}}+\frac{1-t_{2}^{2}}{s_{2}} & -\left(t_{1}+t_{2}\right) .
\end{array}\right)
$$

Equation 106.

So $\operatorname{tr}(T)=\left(t_{1}+t_{2}\right)+\left(-\left(t_{1}+t_{2}\right)\right)=0$. Also, again by Equation 102, we have

$$
\begin{aligned}
\operatorname{tr}\left(T^{2 n+1}\right) & =\operatorname{tr}\left(\left(\varepsilon_{0}+2\right)^{n} T\right) \\
& =\left(\varepsilon_{0}+2\right)^{n} \operatorname{tr}(T) \\
& =0,
\end{aligned}
$$

Equation 107.

for any $n \in \mathbb{N}$.

\section{APPLICATION: $\mathcal{A}_{2}$ AND LUCAS NUMBERS}

Here, as application of the previous sections, we study connections between analytic data obtained from elements of $\mathcal{A}_{2}$ and Lucas numbers. More details about Lucas numbers are found in the references and cited papers thereof. ${ }^{7-11}$

From the main result, Equation 87, of the previous section, we find a functional sequence $\left(f_{n}\right)_{n=1}^{\infty}$, with its $n$-th entries

$$
f_{n}(z)=n \sum_{k=0}^{\left[\frac{n}{2}\right]}(-1)^{k}\left(\frac{n}{k}\right)\left(\begin{array}{c}
n-k-1 \\
k-1
\end{array}\right) z^{n-2 k} .
$$

Equation 108.

for all $n \in \mathbb{N}$, satisfying $\left(X_{1} X_{2}\right)^{n}+\left(\left(X_{1} X_{2}\right)^{n}\right)^{-1}=f_{n}\left(\varepsilon_{0}\right) I_{2} \in \mathcal{A}_{2}$, where $\varepsilon_{0}=\operatorname{det}\left(X_{1}\right)+\operatorname{det}\left(X_{2}\right)-\operatorname{det}\left(X_{1}+X_{2}\right)$.

For any arbitrarily fixed $n \in \mathbb{N}$, consider the summands

$$
\left(\frac{n}{k}\right)\left(\begin{array}{c}
n-k-1 \\
k-1
\end{array}\right), \text { for } k=0,1, \ldots,\left[\frac{n}{2}\right],
$$

Equation 109.

of $f_{n}(z)$.

Define such quantities of Equation 109 by a form of a function, $g: \mathbb{N} \times \mathbb{N}_{0} \rightarrow \mathbb{C}$ by

$$
g(n, k) \stackrel{\text { def }}{=} \begin{cases}\left(\frac{n}{k}\right)\left(\begin{array}{c}
n-k-1 \\
k-1
\end{array}\right) & \text { if } k=0,1, \ldots,\left[\frac{n}{2}\right] \\
0 & \text { otherwise }\end{cases}
$$

Equation 110.

for all $(n, k) \in \mathbb{N} \times \mathbb{N}_{0}$, where $\mathbb{N}_{0} \stackrel{\text { def }}{=} \mathbb{N} \cup\{0\}$. Note that the definition Equation 110 of the function $g$ represents the (non-alternating parts) of summands of $\left(f_{n}\right)_{n=1}^{\infty}$. 
Theorem 18. Let $g$ be a function from $\mathbb{N} \times \mathbb{N}_{0}$ into $\mathbb{C}$ be as described in Equation 110, Then

$$
g(1,0)=1 \text { and } g(n+1, k)=g(n, k)+g(n-1, k-1),
$$

Equation 111.

for all $(n, k) \in(\mathbb{N} \backslash 1) \times \mathbb{N}$.

Proof. Observe first that:

$$
\begin{aligned}
g(n, k) & =\frac{n}{k}\left(\begin{array}{c}
n-k-1 \\
k-1
\end{array}\right) \\
& =\frac{n}{k} \frac{(n-k-1) !}{(k-1) !(n-k-1-k+1) !} \\
& =\frac{n(n-k-1) !}{(n-2 k) ! k !}
\end{aligned}
$$

Equation 112.

whenever $g(n, k)$ is non-zero, for $(n, k) \in \mathbb{N} \times \mathbb{N}_{0}$.

By Equation 112, we obtain that

$$
g(1,0)=\frac{1(1-0-1) !}{(1-0) ! 0 !}=1
$$

Equation 113.

Consider now that:

$$
\begin{aligned}
g(n, k)+g(n-1, k-1) & =\frac{n}{k}\left(\begin{array}{c}
n-k-1 \\
k-1
\end{array}\right)+\frac{n-1}{k-1}\left(\begin{array}{c}
n-1-(k-1)-1 \\
(k-1)-1
\end{array}\right) \\
& =\frac{n(n-k-1) !}{(n-2 k) ! k !}+\frac{(n-1)(n-k-1) !}{(n-2 k+1) !(k-1) !} \\
& =\frac{n(n-2 k+1)(n-k-1) !+k(n-1)(n-k-1) !}{(n-k k+1) ! k !} \\
& =\frac{\left(n^{2}+n k+n-k\right)(n-k-1) !}{(n-2 k+1) ! k !} \\
& =\frac{(n+1)(n-k) !}{k(2 n-2 k+1)(k-1) !} \\
& =\frac{n+1}{k}\left(\begin{array}{c}
(n+1)-k-1 \\
k-1
\end{array}\right),
\end{aligned}
$$

Equation 114.

and hence,

$$
\begin{aligned}
g(n, k)+g(n-1, k-1) & =\frac{n+1}{k}\left(\begin{array}{c}
(n+1)-k-1 \\
k-1
\end{array}\right) \\
& =g(n+1, k) .
\end{aligned}
$$

Equation 115.

Therefore, one can get the recurrence relation Equation 111, by Equation 113 and Equation 115.

By Equation 111, we can see that the family $\left\{g(n, k):(n, k) \in \mathbb{N} \times \mathbb{N}_{0}\right\}$ relates to the following diagram.

By using the above family $\{g(n, k)\}_{(n, k) \in \mathbb{N} \times \mathbb{N}_{0}}$ of quantities obtained from Equation 110, one can re-write the $n$-th entries $f_{n}$ of Equation 108 as follows;

$$
f_{n}(z)=\sum_{k=0}^{\left[\frac{n}{2}\right]}(-1)^{k}(g(n, k)) z^{n-2 k},
$$

Equation 116. 


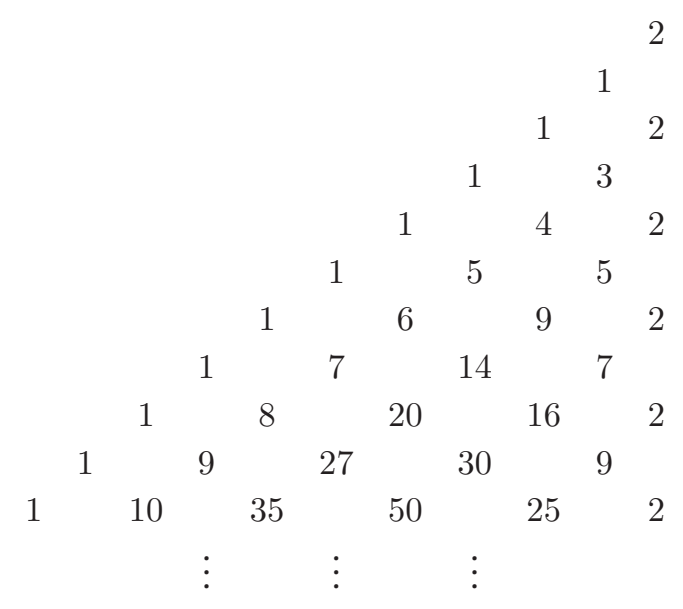

Figure 1. The rows represent $n \in \mathbb{N}$, and the columns represent $k \in \mathbb{N}_{0}$ of the quantities $g(n, k)$. Observe that the diagram is the $L u c a s$ triangle, ${ }^{11}$ induced by the Lucas numbers. ${ }^{7-10}$

for $n \in \mathbb{N}$.

Corollary 19. The coefficients of the functions $f_{n}$ of Equation 108 are determined by the Lucas numbers in the Lucas triangle alternatively.

Moreover, we can verify that

$$
\begin{aligned}
f_{n+1}(z)= & \sum_{k=0}^{\left[\frac{n+1}{2}\right]}(-1)^{k}(g(n+1, k)) z^{n+1-2 k} \\
= & \sum_{k=0}^{\left[\frac{n+1}{2}\right]}(-1)^{k}(g(n, k)+g(n-1, k-1)) z^{n+1-2 k} \\
= & \sum_{k=0}^{\left[\frac{n+1}{2}\right]}(-1)^{k}(g(n, k)) z^{n+1-2 k} \\
& +\sum_{k=1}^{\left[\frac{n+1}{2}\right]}(-1)^{k}(g(n-1, k-1)) z^{n-2 k} \\
= & z\left(\sum_{k=0}^{\left[\frac{n}{2}\right]}(-1)^{k}(g(n, k-1)) z^{n-2 k}\right) \\
& -\sum_{k=0}^{\left[\frac{n-1}{2}\right]}(-1)^{k}(g(n-1, k-1)) z^{n-1-2 k} \\
= & z f_{n}(z)-f_{n-1}(z) .
\end{aligned}
$$

Equation 117.

From the recurrence relation Equation 115, we can re-prove the recurrence relation Equation 78.

Corollary 20. Let

$$
f_{n}(z)=\sum_{k=0}^{\left[\frac{n}{2}\right]}(-1)^{k}(g(n, k)) z^{n-2 k}
$$

Equation 118. 
where $\{g(n, k)\}_{(n, k) \in \mathbb{N} \times \mathbb{N}_{0}}$ are as defined in Equation 110. Then

$$
\begin{aligned}
f_{1}(z) & =z \\
f_{2}(z) & =z^{2}-2 \\
\vdots & \\
f_{n+1}(z) & =z f_{n}(z)-f_{n-1}(z),
\end{aligned}
$$

Equation 119.

for all $n \geq 2$ in $\mathbb{N}$.

Again, the above corollary demonstrates the connection between our Hilbert-space representations of $\Gamma_{2}^{2}$ and Lucas numbers.

\section{REFERENCES}

1. I. Cho, Free Probability on Hecke Algebras and Certain Group $C^{*}$-Algebras Induced by Hecke Algebras, Opuscula Math., (2015) To Appear.

2. I. Cho, Representations and Corresponding Operators Induced by Hecke Algebras, DOI: 10.1007/s11785-014-0418-7, (2014) To Appear.

3. R. Speicher, Combinatorial Theory of the Free Product with Amalgamation and Operator-Valued Free Probability Theory, Momoir, Ame. Math. Soc., 627, (1998)

4. D. Voiculescu, K. J. Dykemma, and A. Nica, Free Random Variables, CRM Monograph Ser., vol.1, ISBN-13: 978-0821811405, (1992)

5. I. Cho, and T. Gillespie, Free Probability on Hecke Algebras, DOI: 10.1007/s11785-014-0403-1, (2014) To Appear.

6. I. Cho, The Moments of Certain Perturbed Operators of the Radial Operator in the Free Group Factors $L\left(F_{N}\right)$, J. Anal. Appl., vol. 5, no.3, (2007) $137-165$.

7. M. Cetin, M. Sezer, and C. Süler, Lucas Polynomial Approach for System of High-Order Linear Differential Equations and Residual Error Estimation, Math. Problems Eng., Article ID: 625984, (2015)

8. A. Nalli, and P. Haukkanem, On Generalized Fibonacci and Lucas Polynomials, Chaos, Solitons \& Fractals, 42, (2009) 3179 - 3186.

9. M. Asci, B. Cekim, and D. Tasci, Generating Matrices for Fibonaci, Lucas and Spectral Orthogonal Polynomials with Algorithms, Internat. J. Pure Appl. Math., 34, no. 2, (2007) 267 - 278.

10. J. Wang, Some New Results for the $(p, q)$-Fibonacci and Lucas Polynomicals, Adv. Diff. Equ., 64, (2014)

11. A. T. Benjamin, The Lucas Triangle Recounted, available at http://www.math.hmc.edu/ benjamin/papers/LucasTriangle.pdf.

\section{ABOUT THE STUDENT AUTHOR}

Ryan Golden is a senior at St. Ambrose University double majoring in Mathematics and Biology with minors in Computer Science and Chemistry. He plans to pursue a PhD in Applied Mathematics, specifically Mathematical and Computational Neuroscience. While his main interests are in the applications of mathematics, he is passionate about theoretical mathematics as well.

\section{PRESS SUMMARY}

In this paper, the authors studied matricial representations of certain finitely presented groups generated by $N$-generators of order-2. As an application, they considered corresponding group algebras under representation. Specifically, they characterized the inverses of all group elements in terms of matrices in the group algebra. From the study of this characterization, the authors realized there are close relations between the trace of the radial operator of the algebras and the Lucas numbers appearing in the Lucas triangle. 